Pacific

Journal of

Mathematics

\title{
DIFFERENTIAL HARNACK AND \\ LOGARITHMIC SOBOLEV INEQUALITIES \\ ALONG RICCI-HARMONIC MAP FLOW
}

ABIMBOLA ABOLARINWA 


\title{
DIFFERENTIAL HARNACK AND LOGARITHMIC SOBOLEV INEQUALITIES ALONG RICCI-HARMONIC MAP FLOW
}

\author{
ABimbola ABOLARINWA
}

\begin{abstract}
This paper introduces a new family of entropy functionals which is proved to be monotonically nondecreasing along the Ricci-harmonic map heat flow. Some of the consequences of the monotonicity are combined to derive gradient estimates and Harnack inequalities for all positive solutions to the associated conjugate heat equation. We relate the entropy monotonicity and the ultracontractivity property of the heat semigroup, and as a result we obtain the equivalence of logarithmic Sobolev inequalities, conjugate heat kernel upper bounds and uniform Sobolev inequalities under Ricci-harmonic map heat flow.
\end{abstract}

\section{Introduction}

Let $(M, g)$ and $(N, \xi)$ be compact Riemannian manifolds (without boundary) of dimensions $m$ and $n$ respectively. Let a smooth map $u: M \rightarrow N$ be a critical point of the Dirichlet energy integral $E(u)=\int_{M}|\nabla u|^{2} d \mu_{g}$, where $N$ is isometrically embedded in $\mathbb{R}^{d}, d \geq n$, by the Nash embedding theorem. The configuration $(g(x, t), u(x, t))$, $t \in[0, T)$, of a one-parameter family of Riemannian metrics $g(x, t)$ and a family of smooth maps $u(x, t)$ is defined to be a Ricci-harmonic map flow if it satisfies the coupled system of nonlinear parabolic equations denoted by $(\mathrm{RH})_{\alpha}$

$$
\left\{\begin{array}{l}
\frac{\partial}{\partial t} g(x, t)=-2 \operatorname{Rc}(x, t)+2 \alpha \nabla u(x, t) \otimes \nabla u(x, t), \\
\frac{\partial}{\partial t} u(x, t)=\tau_{g} u(x, t),
\end{array}\right.
$$

where $\operatorname{Rc}(x, t)$ is the Ricci curvature tensor for the metric $g, \alpha(t) \equiv \alpha>0$ is a time-dependent coupling constant and $\tau_{g} u$ is the intrinsic Laplacian of $u$ which denotes the tension field of the map $u$. The system (1-1) was first studied by B. List [2008] in a special case, $N \subseteq \mathbb{R}$ and $\alpha=2$, where the flow was modified by the Lie derivative of $g$ with respect to a gradient vector field to give a gradient flow

MSC2010: 35J05, 53C44, 58J35, 58J60.

Keywords: Ricci-harmonic map heat flow, monotonicity formula, Harnack inequalities, ultracontractivity, heat semigroup, logarithmic Sobolev inequalities. 
of an energy functional whose stationary points are solutions to the static Einstein vacuum equations arising in general relativity. This has since been generalised by R. Müller [2012] to the general case $N \hookrightarrow \mathbb{R}^{d}$, for sufficiently large $d$. Precisely, the system couples together the Ricci flow of Hamilton [1982] and the heat flow for harmonic maps of Eells and Sampson [1964]. The system (RH) $)_{\alpha}$ is closer to the former in behaviours, such as in existence and singularities, though may be less singular than both. Hence, the analysis of the flow is usually done along the line of Ricci flow and for this, Perelman's works [2002; 2003b; 2003a] on Ricci flow are very applicable to the theory and applications of the Ricci-harmonic map flow.

In this paper we study the behaviour of all positive solutions to the associated conjugate heat equation along the Ricci-harmonic map flow. Let $h, H: M \times[0, T) \rightarrow$ $(0, \infty)$ satisfy

$$
\left(\frac{\partial}{\partial t}-\Delta_{g}\right) h=0 \quad \text { and } \quad\left(-\frac{\partial}{\partial t}-\Delta_{g}+R-\alpha|\nabla u|_{g}^{2}\right) H=0,
$$

with

$$
\int_{0}^{T} \int_{M}\left(\frac{\partial}{\partial t}-\Delta_{g}\right) h H d \mu_{g} d t=\int_{0}^{T} \int_{M} h\left(-\frac{\partial}{\partial t}-\Delta_{g}+R-\alpha|\nabla u|_{g}^{2}\right) H d \mu_{g} d t,
$$

where $\Delta_{g}$ is the usual Laplace-Beltrami operator and $\square^{*}:=-\partial / \partial t-\Delta_{g}+R-$ $\alpha|\nabla u|_{g}^{2}$ is the standard conjugate to the heat operator $\square:=\partial / \partial t-\Delta_{g}$. We say $h$ and $H$ are respectively solutions to the heat equation and conjugate heat equation. The main idea here is to solve the Ricci-harmonic map flow forward in time and solve the conjugate heat equation backward in time. Fixing the coordinate $(y, s)$, $H=H(x, t ; y, s)$ will be called the conjugate heat kernel (the positive minimal solution) if it tends to a $\delta$-function as $t \rightarrow T$.

Our main results in the first part of this paper are Perelman's differential Harnack estimates for $f \in C^{\infty}(M \times[0, T))$ satisfying $H(x, \tau ; y, s)=(4 \pi \tau)^{-m / 2} e^{-f(x, \tau)}$, $\tau=T-t$,

$$
-\frac{d}{d t} f(\gamma(\tau), \tau) \leq \frac{1}{2}\left(\left|\gamma^{\prime}(\tau)\right|^{2}+S_{g}(\gamma(\tau), \tau)-\frac{m}{2 \tau}\right),
$$

and $\mathrm{Li}-\mathrm{Yau}$ Harnack estimates for all positive solutions to the conjugate heat equation

$$
\frac{H\left(x_{2}, t_{2}\right)}{H\left(x_{1}, t_{1}\right)} \leq\left(\frac{\tau_{1}}{\tau_{2}}\right)^{m / 4} \exp \left(\frac{1}{2} \int_{t_{1}}^{t_{2}}\left(\left|\gamma^{\prime}(t)\right|^{2}+S_{g}(\gamma(t), t) d t\right),\right.
$$

where $S_{g}=R_{g}-\alpha|\nabla u|_{g}^{2}$. (The proofs of (1-2) and (1-3) are delayed until Section 4). Both results stated above are consequences of a monotonicity formula for a new entropy functional $\mathcal{W}_{\alpha, \epsilon}$ introduced in Section 3, where we obtain the Harnack 
inequality for $0<\epsilon^{2} \leq 4 \pi$,

$$
\frac{\epsilon^{2} \tau}{4 \pi}\left(2 \Delta f-|\nabla f|^{2}+S_{g}\right)+f-\frac{m \epsilon^{2}}{4 \pi} \leq 0
$$

for all time $t$ and prove that

$$
\lim _{\tau \rightarrow 0} \int_{M} \frac{\epsilon^{2} \tau}{4 \pi}\left(2 \Delta f-|\nabla f|^{2}+S_{g}\right)+f-\frac{m \epsilon^{2}}{4 \pi} h H d \mu_{g} \longrightarrow 0,
$$

with the condition that $\epsilon^{2} \rightarrow 4 \pi$ as $t \rightarrow T$. Monotonicity formulas are generally useful in controlling solutions of evolution equations. This entropy is also intimately related to the logarithmic Sobolev inequality of Gross [1975]. Perelman used this property to obtain upper bounds for the fundamental solution to the adjoint heat equation via his reduced length. This leads to the proof of the noncollapsing theorem on Riemannian manifolds and, consequently, to the completion of R. Hamilton's program on the Poincaré conjecture. See [Perelman 2002; 2003b; 2003a; Cao et al. 2003]. Among several examples, Perelman's entropy and the gradient estimates of Li and Yau [1986] are important ones that show close relations between entropy monotonicity and the gradient estimate for the heat equation (forward or backward in time). Lei Ni [2004] has also considered a case for the linear heat equation on a static manifold with nonnegative Ricci curvature. We notice that coupling a heat-type equation with geometric flow began with [Hamilton 1993] and it has since become a very active research area and has led to numerous physical and geometric applications; for examples, see [Băileșteanu et al. 2010; Băileşteanu and Tran 2013; Cao and Zhang 2011; Kuang and Zhang 2008; List 2008; Müller 2012; Ni 2006; Zhang 2007] and the references therein.

Another important application of Perelman's $\mathcal{W}$-entropy monotonicity is in the derivation of uniform Sobolev inequalities by Q. Zhang [2007]; see also [Hsu 2008; Ye 2007]. In the second part of this paper, we relate the entropy monotonicity and the ultracontractivity property of the heat semigroup, and as a result we establish the equivalence of logarithmic Sobolev inequalities, conjugate heat kernel upper bounds and uniform Sobolev inequalities under Ricci-harmonic map heat flow. Precisely, let $A_{0}$ and $B_{0}$ be finite positive constants depending only on $m, g_{0}$, the lower bound for the Ricci curvature and the injectivity radius of $M$. For any $v \in W^{1,2}\left(M, g_{0}\right)$ such that

$$
\|v\|_{2 m /(m-2)} \leq A_{0}\|\nabla v\|_{2}+B_{0}\|v\|_{2},
$$

where $m \geq 3$ and $\|\cdot\|_{q}=\left(\int_{M}|\cdot|^{q} d \mu_{g}\right)^{1 / q}, 1 \leq p<\infty$, we have the following result.

Theorem. Let $M$ be a compact Riemannian manifold of dimension $m \geq 3$. Let the solution to the $(\mathrm{RH})_{\alpha}$-flow exist for all times $t \in[0, T)$. Assume the Sobolev 
embedding (1-6) holds; then for finite positive constants $A$ and $B$ depending on $m$, $A_{0}, B_{0}$, the lower bound for $R_{g_{0}}$ and $T$,

$$
\left(\int_{M} v^{2 m /(m-2)} d \mu_{g}\right)^{(m-2) / 2} \leq A \int_{M}\left(|\nabla v|^{2}+\frac{1}{4} S_{g} v^{2}\right) d \mu_{g}+B \int_{A} v^{2} d \mu_{g}
$$

and

$$
\begin{aligned}
\int_{M} v^{2} & \ln v^{2} d \mu_{g(t)} \\
& \leq \sigma^{2} \int_{M}\left(4|\nabla v|^{2}+S_{g} v^{2}\right) d \mu_{g(t)}-\frac{m}{2} \ln \sigma^{2}+\left(t+\sigma^{2}\right) \beta_{1}+\frac{m}{2} \ln \frac{m A}{2 e},
\end{aligned}
$$

hold for each $t \in[0, T)$ and $v \in W^{1,2}(M)$ if $\lambda_{\alpha}=\inf _{\|v\|_{2}=1} \int_{M}\left(4|\nabla v|^{2}+S_{g} v^{2}\right) d \mu_{g_{0}}$; that is, $\lambda_{\alpha 0}$ is the first eigenvalue of the operator $-\Delta+\frac{1}{4} S_{g}$.

Finally, for some constant $C$ depending on $m, t, T, A_{0}, B_{0}$ and $\sup S_{g}(\cdot, 0)$, the estimate

$$
H(x, T ; y) \leq C T^{-m / 2}
$$

for the positive solution to the conjugate heat equation associated to $(\mathrm{RH})_{\alpha}$ holds.

The three results in the above theorem are essentially equivalent, and their proofs occupy Sections $5-7$. The approach to the proof here is Sobolev inequality (1-7) $\Longrightarrow$ log-Sobolev inequality (1-8) $\Longrightarrow$ heat kernel upper bound (1-9) $\Longrightarrow$ Sobolev inequality (1-7). Indeed, any of them can be derived from the other. The results of the above form [Hsu 2008; Ye 2007; Zhang 2007] yield a long time $\kappa$-noncollapsing estimate which generalises Perelman's short time result [2002] along the Ricci flow.

We recall that the nonnegativity of the scalar curvature $R_{g}$ is preserved along Ricci flow [Chow and Knopf 2004], so the nonnegativity of $S_{g}$ is also preserved as long as $(\mathrm{RH})_{\alpha}$ exists. Indeed, $S_{g}$ evolves by a reaction-diffusion equation which helps to visualise its behaviour up to singular time (we discuss this in the next section). The condition $S_{g}=R_{g}-\alpha|\nabla u|_{g}^{2} \geq 0$ at the starting time $t=0$ must now be considered. The assumption is not necessary for the derivation of (1-7) since additional geometric data are not usually required to derive a Sobolev inequality from either a log-Sobolev inequality or the heat kernel bound. The assumption is required for the condition that a certain eigenvalue $\lambda_{\alpha}$ for the initial metric is positive, which is required to pass to (1-8). The class of manifold $\left(M, g_{0}\right)$ with $\lambda_{\alpha} 0>0$ is a very large one and significant from a geometric point of view. Moreover, if $\lambda_{\alpha} 0>0$ for $S_{g}(0)>0$ (i.e., $R_{g}(0)>\alpha(0)|\nabla u(0)|^{2}$ ) then $A, B$ are independent of time and $B=0$. Corollary 7.5 below presents corresponding versions of (1-7) and (1-8) in this case.

In the next section we discuss necessary background on Perelman-Müller entropy monotonicity formulas for $(\mathrm{RH})_{\alpha}$. 


\section{Background on entropy formulas for $(\mathrm{RH})_{\alpha}$-flow}

Let $(M, g)$ be a compact Riemannian manifold. For the metric $g$, any smooth functions $u \in C^{\infty}(M, N), u(M) \subseteq N \hookrightarrow \mathbb{R}^{n}, f \in C^{\infty}(M)$ and constant $\alpha>0$, Perelman and Müller's energy functional [Müller 2012] is defined on the triple $(g, u, f)$ by

$$
\mathcal{F}_{\alpha}(g, u, f):=\int_{M}\left(R_{g}+|\nabla f|_{g}^{2}-\alpha|\nabla u|_{g}^{2}\right) e^{-f} d \mu_{g},
$$

which can also be written in two other ways,

$$
\begin{aligned}
\mathcal{F}_{\alpha}(g, u, f) & =\int_{M}\left(S_{g}+\Delta_{g} f\right) e^{-f} d \mu_{g} \\
& =\int_{M}\left(2 \Delta_{g} f-|\nabla f|_{g}^{2}+S_{g}\right) e^{-f} d \mu_{g},
\end{aligned}
$$

since $\int_{M} \Delta\left(e^{-f}\right)=0=\int_{M}\left(-\Delta f+|\nabla f|_{g}^{2}\right) e^{-f} d \mu_{g}$. For any diffeomorphism $\phi: M \rightarrow M$, we have $\mathcal{F}_{\alpha}\left(\phi^{*} g, \phi^{*} u, \phi^{*} f\right)=\mathcal{F}_{\alpha}(g, u, f)$. If $(g, u)$ is a solution to the system (1-1), Müller [2012] proved that the $\mathcal{F}_{\alpha}$-functional is nondecreasing under the flow and showed that the system is equivalent (after pulling back with a diffeomorphism generated by a vector field) to the gradient flow system for the energy functional $\mathcal{F}_{\alpha}$, locally written as,

$$
\left\{\begin{array}{l}
\frac{\partial}{\partial t} g_{i j}=-2 R_{i j}+2 \alpha \nabla_{i} u \otimes \nabla_{j} u+2 \nabla_{i} \nabla_{j} f, \\
\frac{\partial}{\partial t} u=\tau_{g} u-\langle\nabla u, \nabla f\rangle, \\
\frac{\partial}{\partial t} f=-R+\alpha|\nabla u|^{2}-\Delta f .
\end{array}\right.
$$

More precisely,

$$
\begin{aligned}
& \frac{d}{d t} \mathcal{F}_{\alpha}(g, u, f) \\
& \quad=2 \int_{M}\left(|\operatorname{Rc}-\alpha \nabla u \otimes \nabla u+\nabla \nabla f|^{2}+\alpha\left|\tau_{g} u-\langle\nabla u, \nabla f\rangle\right|^{2}\right) e^{-f} d \mu_{g} \geq 0 .
\end{aligned}
$$

An application of this is that $\mathcal{F}_{\alpha}$ is constant if and only if $(g, u)$ is a steady gradient soliton.

Define

$$
\lambda_{\alpha}(g)=\inf \left\{\mathcal{F}_{\alpha}(g, u, f): f \in C^{\infty}(M), \int_{M} e^{-f} d \mu_{g}=1\right\} .
$$

Then $\lambda_{\alpha}(g)$ is the first eigenvalue of the operator $-4 \Delta+S_{g}$, where the nondecreasing property of $\mathcal{F}_{\alpha}$ implies $\lambda_{\alpha}(g)$ is nondecreasing and we have, by setting $v=e^{-f / 2}$, 
the corresponding normalised eigenvector,

$$
-4 \Delta v+S_{g} v=\lambda_{\alpha}(g) v .
$$

Hence

$$
\lambda_{\alpha}(g, u)=\inf \left\{\int_{M}\left(4|\nabla v|^{2}+S_{g} v^{2}\right) d \mu_{g}: \int_{M} v^{2} d \mu_{g}=1\right\} .
$$

Similar to the case of Hamilton's Ricci flow, all geometric quantities associated with the source manifold evolve along $(\mathrm{RH})_{\alpha}$. For instance, we consider those quantities that are directly relevant at the present; the metric inverse, volume element, Laplace-Beltrami operator and $S_{g}$ evolve as follows:

$$
\begin{aligned}
\frac{\partial}{\partial t} g^{i j} & =2 S^{i j}, & \frac{\partial}{\partial t} \Delta_{g} & =2 S^{i j} \nabla_{i} \nabla_{j}-2 \alpha \tau_{g} u\langle\nabla u, \nabla \cdot\rangle, \\
\frac{\partial}{\partial t} d \mu_{g} & =-S_{g} d \mu_{g}, & \frac{\partial}{\partial t} S_{g} & =\Delta S_{g}+2\left|S_{i j}\right|^{2}+2 \alpha\left|\tau_{g} u\right|_{g}^{2},
\end{aligned}
$$

where $S_{i j}=\mathrm{Rc}-\alpha \nabla u \otimes \nabla u$ and $g^{i j} S_{i j}=S_{g}$. The nonnegativity of the curvature operator and $S_{g}$ are preserved during the flow; for example, the evolution of $S_{g}=$ $R_{g}-\alpha|\nabla u|^{2}$ is governed by the differential inequality

$$
\frac{\partial}{\partial t} S_{g} \geq \Delta S_{g}+\frac{2}{m} S_{g}^{2}
$$

since $\alpha \geq 0$ and $\left|S_{i j}\right|^{2} \geq(1 / m) S_{g}^{2}$. Suppose $S_{g_{0}} \geq \rho$. We can use the maximum principle by comparing the solution of the above inequality with that of the ODE

$$
\left\{\begin{array}{l}
\frac{d \psi(t)}{d t}=\frac{2}{m}(\psi(t))^{2}, \\
\psi(0)=\rho,
\end{array}\right.
$$

solving to

Therefore,

$$
\psi(t)=\frac{1}{\frac{1}{\rho}-\frac{2}{m} t} .
$$

$$
S_{g(t)} \geq \psi(t)=\frac{1}{\frac{1}{\rho}-\frac{2}{m} t}
$$

for all $t \geq 0$ as long as the flow exists. We remark that (2-5) implies

$$
S_{g(t) \min } \geq \frac{S_{g(0) \min }}{1-(2 t / m) S_{g(0) \min }} .
$$

Clearly, $S_{g(0) \min }>0$ implies $S_{g(t) \min } \rightarrow \infty$ in finite time $T_{\epsilon} \leq m /\left(2 S_{g(0) \min }\right)<\infty$. This also implies that $R_{g(t) \min } \rightarrow \infty$ as $t \rightarrow T_{\epsilon}$, and thus $g(t)$ becomes singular in finite time $T_{\text {singular }} \leq T_{\epsilon}<\infty$. 
Recall the Perelman-Müller $\mathcal{W}_{\alpha}$-entropy functional also introduced in [Müller 2012] as

$$
\mathcal{W}_{\alpha}(g, u, f, \tau):=\int_{M}\left(\tau\left(S_{g}+|\nabla f|_{g}^{2}\right)+f-m\right) \frac{e^{-f}}{(4 \pi \tau)^{m / 2}} d \mu_{g},
$$

where $\tau \in \mathbb{R}$ is a real number, $f \in C^{\infty}(M \times[0, T)), \alpha>0$ is a constant and $u \in C^{\infty}(M, N)$ is a harmonic map between the $m$-dimensional manifold $M$ and the $n$-dimensional manifold $N$, which by the Nash embedding theorem is isometrically embedded in $\mathbb{R}^{d}$ for sufficiently large $d$. The above entropy functional is analogous to Perelman's $\mathcal{W}$-entropy for shrinkers [2002] under the Ricci flow. $\mathcal{W}_{\alpha}$ is equally used for shrinkers under Ricci-harmonic map flow as can be traced back to List [2008]. As pointed out in [Perelman 2002], such an entropy is invariant and monotone. In fact, given a constant $\lambda>0$ and a diffeomorphism $\phi$ of $M$, under simultaneous scaling of $g$ and $\tau$, we have

$$
\mathcal{W}_{\alpha}(\lambda g, u, f, \lambda \tau)=\mathcal{W}_{\alpha}(g, u, f, \tau),
$$

and under the pullback of $g, u$ and $f$, we have

$$
\mathcal{W}_{\alpha}\left(\phi^{*} g, \phi^{*} u, \phi^{*} f, \tau\right)=\mathcal{W}_{\alpha}(g, u, f, \tau) .
$$

More importantly, we have the following monotonicity formula.

Proposition 2.1 [List 2008; Müller 2012]. Let $(g(t), u(t), f(t), \tau(t)), t \in[0, T)$ be a solution of the system

$$
\left\{\begin{array}{l}
\frac{\partial}{\partial t} g=-2 \mathrm{Rc}+2 \alpha \nabla u \otimes \nabla u \\
\frac{\partial}{\partial t} u=\tau_{g} u \\
\left(-\frac{\partial}{\partial t}-\Delta_{g}+R-\alpha|\nabla u|_{g}^{2}\right) \frac{e^{-f}}{(4 \pi \tau)^{m / 2}}=0, \\
\frac{\partial}{\partial t} \tau=-1 .
\end{array}\right.
$$

Then the $\mathcal{W}_{\alpha}$-entropy is nondecreasing with

$$
\begin{aligned}
\frac{d}{d t} \mathcal{W}_{\alpha}(g, u, f, \tau)=2 \tau \int_{M} \mid \mathrm{Rc} & -\alpha \nabla u \otimes \nabla u+\nabla \nabla f-\left.\frac{1}{2 \tau} g\right|^{2} \frac{e^{-f}}{(4 \pi \tau)^{m / 2}} d \mu_{g} \\
+ & 2 \tau \int_{M} \alpha\left|\tau_{g} u-\langle\nabla u, \nabla f\rangle\right|^{2} \frac{e^{-f}}{(4 \pi \tau)^{m / 2}} d \mu_{g} .
\end{aligned}
$$

Notice that the third equation in the above system is equivalent to the following backward heat equation

$$
\frac{\partial f}{\partial t}=-\Delta_{g} f+|\nabla f|_{g}^{2}-R+\alpha|\nabla u|_{g}^{2}+\frac{m}{2 \tau}
$$


A monotonicity formula of the above type is used to rule out any periodic solution to the $(\mathrm{RH})_{\alpha}$-flow other than those that are striking and Einstein [List 2008; Müller 2012; Perelman 2002].

Similar to $\lambda_{\alpha}(g, u)$ above, define the minimizing problem

$$
\mu_{\alpha}(g, u, \tau):=\inf \left\{\mathcal{W}_{\alpha}(g, u, f, \tau): f \in C^{\infty}(M), \int_{M}(4 \pi \tau)^{-m / 2} e^{-f} d \mu_{g}=1\right\},
$$

replacing $f$ by $v=e^{-f / 2}$. We have an equivalent minimizing integral

$$
\mathcal{W}_{\alpha}(g, u, v, \tau)=\int_{M}\left(\tau\left(4|\nabla v|^{2}+S_{g} v^{2}\right)-v^{2} \ln v^{2}-m v^{2}\right)(4 \pi \tau)^{-m / 2} d \mu_{g}
$$

for functions $v \in H^{1}(M)$ with $\int_{M} v^{2}(4 \pi \tau)^{-m / 2} d \mu_{g}=1$. Then $v$ satisfies the EulerLagrange equation, and it follows that $\mu_{\alpha}(g, u, \tau)$ is achieved by a minimizer $f_{\tau}$ satisfying

$$
\tau\left(2 \Delta f_{\tau}-\left|\nabla f_{\tau}\right|^{2}+S_{g}\right)+f_{\tau}-n=\mu(g, \tau) .
$$

By the result of Perelman, it is well understood that for any metric $g$ on a compact manifold $M$ and $\tau>0$, we have $\mu(g, u, \tau)>-\infty$ and it approaches zero as $\tau \rightarrow 0$.

\section{A new entropy monotonicity formula}

In this section we introduce a new family of dual entropy formulas, which are dual in the sense that they generalise Ni's entropy formula [2004] for the forward heat equation on the one hand and generalise Perelman and Müller's $\mathcal{W}_{\alpha}$-entropy on the other hand. A similar family of entropy functionals was constructed by Kuang and Zhang [2008]. The monotonicity property discussed here is very crucial to the derivation of our results in the rest of this paper.

Definition 3.1. Let $f: M \times[0, T] \rightarrow \mathbb{R}$ be smoothly defined with normalisation condition

$$
\int_{M} \frac{e^{-f}}{(4 \pi \tau)^{m / 2}} d \mu_{g}=1
$$

We define a generalised family of entropy by

$\mathcal{W}_{\alpha, \epsilon}(g, u, f, \tau)=\int_{M}\left(\frac{\epsilon^{2} \tau}{4 \pi}\left(S_{g}+|\nabla f|_{g}^{2}\right)+f-\frac{m \epsilon^{2}}{4 \pi}+\frac{m}{2} \ln \frac{4 \pi}{\epsilon^{2}}\right) \frac{e^{-f}}{(4 \pi \tau)^{m / 2}} d \mu_{g}$,

where $\tau(t)=T-t>0,0<\epsilon^{2} \leq 4 \pi$ and $S_{g}=S_{g}(x, t)=\left(R_{g}-\alpha|\nabla u|_{g}^{2}\right)(x, t)$.

Let $H=H(x, t)$ be a positive solution to the conjugate heat equation on a complete compact manifold with metric $g=g(x, t)$, evolving by the $(\mathrm{RH})_{\alpha}$. Let 
$H=(4 \pi \tau)^{-m / 2} e^{-f}$ and $\int_{M} H d \mu_{g}=1$. Then

$$
\left(-\frac{\partial}{\partial t}-\Delta_{g}+S_{g}\right) H=0 .
$$

Theorem 3.2. Suppose that $(g(t), u(t)), t \in[0, T)$, solves $(\mathrm{RH})_{\alpha}$ with $\alpha(t) \equiv \alpha>0$ and $\tau$ is a backward time with $\partial \tau / \partial t=-1$. Suppose that $H: M \times[0, T) \rightarrow(0, \infty)$ solves the conjugate heat equation $\left(-\partial / \partial t-\Delta_{g}+S_{g}\right) H=0$. The entropy functional $\mathcal{W}_{\alpha, \epsilon}$ is nondecreasing by the formula

$$
\begin{aligned}
& \frac{d}{d t} \mathcal{W}_{\alpha, \epsilon}(g, u, f, \tau) \\
& \geq \frac{\epsilon^{2} \tau}{2 \pi} \int_{M}\left(\left|\mathrm{Rc}-\alpha \nabla u \otimes \nabla u+\nabla \nabla f-\frac{1}{2 \tau} g\right|^{2}+\alpha\left|\tau_{g} u-\langle\nabla u, \nabla f\rangle\right|^{2}\right) H d \mu_{g}
\end{aligned}
$$

for $0<\epsilon^{2} \leq 4 \pi$.

Remark 3.3. We remark that if $\epsilon^{2}=4 \pi$, we recover Perelman and Müller's $\mathcal{W}_{\alpha}$-entropy.

Scaling and diffeomorphism invariance of $\mathcal{W}_{\alpha, \epsilon}$. Before we prove the monotonicity formula (3-3), we shall first establish the invariance of our new entropy with respect to dilation and diffeomorphism.

Lemma 3.4 [Chow and Knopf 2004, Lemma 6.57]. If $g$ and $h$ are two Riemannian metrics on an n-dimensional Riemannian manifold and they are related by the time-scale factor $\lambda$ (i.e., $g=\lambda h$ ), then the various geometric quantities scale as follows:

$$
\begin{gathered}
g^{i j}=\frac{1}{\lambda} h^{i j}, \quad \Gamma_{i j(g)}^{k}=\Gamma_{i j(h)}^{k}, \\
R_{i j k}^{l}(g)=R_{i j k}^{l}(h), \quad R_{i j k l}(g)=\phi R_{i j k l}(h), \\
R_{i j}(g)=R_{i j}(h), \quad R_{(g)}=\frac{1}{\lambda} R_{(h)}, \quad d \mu_{(g)}=\lambda^{n / 2} d \mu_{(h)} .
\end{gathered}
$$

Lemma 3.5. Let $\lambda>0$ be any constant and $\phi: M \rightarrow M$ be a one-parameter family of diffeomorphisms. Then

$$
\begin{aligned}
\mathcal{W}_{\alpha, \epsilon}(\lambda g, u, f, \lambda \tau) & =\mathcal{W}_{\alpha, \epsilon}(g, u, f, \tau), \\
\mathcal{W}_{\alpha, \epsilon}\left(\phi^{*} g, \phi^{*} u, \phi^{*} f, \tau\right) & =\mathcal{W}_{\alpha, \epsilon}(g, u, f, \tau) .
\end{aligned}
$$

Proof. By a straightforward computation, we have

$$
\begin{array}{r}
\mathcal{W}_{\alpha, \epsilon}(\lambda g, u, f, \lambda \tau)=\int_{M}\left(\frac{\epsilon^{2} \lambda \tau}{4 \pi}\left(R(\lambda g)-\alpha(\lambda g)^{i j} \nabla_{i} u \otimes \nabla_{j} u+(\lambda g)^{i j} \nabla_{i} f \nabla_{j} f\right)\right. \\
\left.+f-\frac{m \epsilon^{2}}{4 \pi}+\frac{m}{2} \ln \frac{4 \pi}{\epsilon^{2}}\right) \frac{e^{-f}}{(4 \pi \lambda \tau)^{m / 2}} \sqrt{\operatorname{det}(\lambda g)} d x
\end{array}
$$




$$
\begin{aligned}
& =\int_{M}\left(\frac{\epsilon^{2} \lambda \tau}{4 \pi}\left(\lambda^{-1} R(g)-\alpha \lambda^{-1} g^{i j} \nabla_{i} u \otimes \nabla_{j} u+\lambda^{-1} g^{i j} \nabla_{i} f \nabla_{j} f\right)\right. \\
& \left.\quad+f-\frac{m \epsilon^{2}}{4 \pi}+\frac{m}{2} \ln \frac{4 \pi}{\epsilon^{2}}\right) \frac{e^{-f}}{\lambda^{m / 2}(4 \pi \tau)^{m / 2}} \sqrt{\lambda^{m} \operatorname{det}(g)} d x \\
& =\int_{M}\left(\frac{\epsilon^{2} \tau}{4 \pi}\left(R_{g}-\alpha|\nabla u|_{g}^{2}+|\nabla f|_{g}^{2}\right)\right. \\
& \left.\quad+f-\frac{m \epsilon^{2}}{4 \pi}+\frac{m}{2} \ln \frac{4 \pi}{\epsilon^{2}}\right) \frac{e^{-f}}{(4 \pi \tau)^{m / 2}} d \mu_{g} \\
& =\mathcal{W}_{\alpha, \epsilon}(g, u, f, \tau) .
\end{aligned}
$$

The invariance under diffeomorphisms is trivial since $(\mathrm{RH})_{\alpha}$-flow is equivalent to the flow modified by the time-dependent diffeomorphism $\phi$ generated by the gradient of $f$, where $\phi^{*} g$ is the pulled-back metric and $\phi^{*} f=f \circ \phi$. For the harmonic map $u$, the invariance holds if we combine the following facts: $\phi$ is a $C^{\infty}$-diffeomorphism and $u \in C^{\infty}(M, N)$ is a harmonic map with respect to $(M, g)$; then $\phi^{*} u=u \circ \phi \in C^{\infty}(M, N)$ is a harmonic map with respect to $\left(M, \phi^{*} g\right)$ with the identity

$$
\int_{M}|\nabla u|_{g}^{2} d \mu_{g}=\int_{M}|\nabla(u \circ \phi)|_{\phi^{*} g}^{2} d \mu_{\phi^{*} g} .
$$

Then, all the geometric quantities are invariant under $(\mathrm{RH})_{\alpha}$-flow and the diffeomorphism invariance of $\mathcal{W}_{\alpha, \epsilon}$ follows.

\section{Proof of Theorem 3.2 (the monotonicity formula for $\mathcal{W}_{\alpha, \epsilon}$ ).}

Proof. The entropy functional can be rewritten as

$\mathcal{W}_{\alpha, \epsilon}(g, u, f, \tau)$

$=\frac{\epsilon^{2}}{4 \pi} \int_{M}\left(\tau\left(S_{g}+|\nabla f|^{2}\right)+f-m\right) H d \mu_{g}+\left(1-\frac{\epsilon^{2}}{4 \pi}\right) \int_{M} f H d \mu_{H}+\frac{m}{2} \ln \frac{4 \pi}{\epsilon^{2}}$.

By direct computation we obtain the evolution equation

(3-4) $\frac{d}{d t} \mathcal{W}_{\alpha, \epsilon}(g, u, f, \tau)=\frac{\epsilon^{2}}{4 \pi} \frac{\partial}{\partial t}\left(\int_{M} V d \mu_{g}\right)+\left(1-\frac{\epsilon^{2}}{4 \pi}\right) \frac{\partial}{\partial t}\left(\int_{M} f H d \mu_{g}\right)$, where

$$
V:=\left(\tau\left(2 \Delta_{g} f+S_{g}-|\nabla f|^{2}\right)+f-m\right) H
$$

since $\int_{M}\left(\Delta_{g} f-|\nabla f|_{g}^{2}\right) e^{-f} d \mu_{g}=0$ on a closed manifold $M$. We make two claims here, which we shall prove in the next two propositions, namely,

$$
\frac{\partial}{\partial t}\left(\int_{M} V d \mu_{g}\right)=\int_{M}-\square^{*} V d \mu_{g}=\frac{d}{d t} \mathcal{W}_{\alpha}(g, u, f, \tau)
$$


and

$$
\frac{\partial}{\partial t}\left(\int_{M} f H d \mu_{g}\right)=-\mathcal{F}_{\alpha}(g, u, f)+\frac{m}{2 \tau} \geq 0 .
$$

With the above two claims, we arrive at

$$
\frac{d}{d t} \mathcal{W}_{\alpha, \epsilon}(g, u, f, \tau)=\frac{\epsilon^{2}}{4 \pi} \frac{d}{d t} \mathcal{W}_{\alpha}(g, u, f, \tau)+\frac{m}{2 \tau}-\mathcal{F}_{\alpha}(g, u, f),
$$

which proves the monotonicity formula (3-3) for $0<\epsilon^{2} \leq 4 \pi$.

Proposition 3.6. With the assumptions of Theorem 3.2, the quantity

$$
V:=\left(\tau\left(2 \Delta_{g} f+S_{g}-|\nabla f|^{2}\right)+f-m\right) H
$$

satisfies

(3-9) $\square^{*} V=-2 \tau\left(\left|\mathrm{Rc}-\alpha \nabla u \otimes \nabla u+\nabla \nabla f-\frac{1}{2 \tau} g\right|^{2}+\alpha\left|\tau_{g} u-\langle\nabla u, \nabla f\rangle\right|^{2}\right) H$

and

$$
\frac{d}{d t} \mathcal{W}_{\alpha}(g, u, f, \tau)=-\int_{M} \square^{*} V d \mu_{g}
$$

Moreover if $H$ tends to a $\delta$-function as $t \rightarrow T$, then $V \leq 0$ for all $t<T$ with $H(x, \tau)$ replaced with $H(x, \tau ; y, \sigma)$, the fundamental solution.

Proof. Let $P=\tau\left(2 \Delta f-|\nabla f|^{2}+S_{g}\right)+f-n$, and $\partial_{t} \tau=-1$ since $\tau=T-t$. Thus,

$$
\begin{aligned}
\square^{*} V & =\left(-\partial_{t}-\Delta+S_{g}\right)(P H) \\
& =-\left(\partial_{t}+\Delta\right) P H-2\langle\nabla P, \nabla H\rangle
\end{aligned}
$$

and

$$
H^{-1} \square^{*} V=-\left(\partial_{t}+\Delta\right) P+2\langle\nabla P, \nabla f\rangle
$$

since $f=-\ln H-(m / 2) \ln (4 \pi \tau)$ implies that $\nabla f=-H^{-1} \nabla H$. Let us compute $\left(\partial_{t}+\Delta\right) P$ as follows:

$$
\frac{\partial P}{\partial t}=-\left(2 \Delta f-|\nabla f|^{2}+S_{g}\right)+\tau \frac{\partial}{\partial t}\left(2 \Delta f-|\nabla f|^{2}\right)+\tau \frac{\partial}{\partial t} S_{g}+\frac{\partial}{\partial t} f .
$$

Note that

$$
\begin{gathered}
\partial_{t} f=-\Delta_{g} f-S_{g}+|\nabla f|_{g}^{2}+\frac{m}{2 \tau}, \\
\partial_{t} S_{g}=\Delta S_{g}+2\left|S_{i j}\right|_{g}^{2}+2 \alpha\left|\tau_{g} u\right|_{g}^{2} .
\end{gathered}
$$

Then a straightforward computations yields

$$
\begin{aligned}
& 2 \frac{\partial}{\partial t}(\Delta f)=4 S_{i j} \nabla_{i} \nabla_{j} f-4 \alpha \tau_{g} u\langle\nabla u, \nabla f\rangle+2 \Delta\left(-\Delta f+|\nabla f|^{2}-S_{g}\right), \\
& \frac{\partial}{\partial t}|\nabla f|^{2}=\frac{\partial}{\partial t}\left(g^{i j} \nabla_{i} f \nabla_{j} f\right)=2 S_{i j} \nabla_{i} f \nabla_{j} f+2\left\langle\nabla f, \nabla \frac{\partial}{\partial t} f\right\rangle .
\end{aligned}
$$


Combining (3-11)-(3-13) with the identity $\Delta P=\tau\left(2 \Delta(\Delta f)-\Delta|\nabla f|^{2}+\Delta S_{g}\right)+\Delta f$, we have

$$
\begin{aligned}
\left(\frac{\partial}{\partial t}+\Delta\right) P= & -2 \Delta f-2|\nabla f|^{2}-2 S_{g}+\frac{m}{2 \tau} \\
+ & \tau\left(4 S_{i j} \nabla_{i} \nabla_{j} f-4 \alpha \tau_{g} u\langle\nabla u, \nabla f\rangle+\Delta|\nabla f|^{2}+2\langle\nabla f, \nabla \Delta f\rangle\right. \\
& \left.-2 S_{i j} \nabla_{i} f \nabla_{j} f+2\left|S_{i j}\right|^{2}+2 \alpha\left|\tau_{g} u\right|^{2}-2\left\langle\nabla f, \nabla|\nabla f|^{2}\right\rangle+2\left\langle\nabla f, \nabla S_{g}\right\rangle\right) .
\end{aligned}
$$

Similarly,

$$
\begin{aligned}
2\langle\nabla P, \nabla f\rangle & =2\left\langle\nabla\left(\tau\left(2 \Delta f-|\nabla f|^{2}+S-g\right)+f\right), \nabla f\right\rangle \\
& =2 \tau\left(2\langle\nabla \Delta f, \nabla f\rangle-\left\langle\nabla|\nabla f|^{2}, \nabla f\right\rangle+\left\langle\nabla S_{g}, \nabla f\right\rangle\right)+2|\nabla f|^{2} .
\end{aligned}
$$

Therefore,

$$
\begin{aligned}
& -\left(\frac{\partial}{\partial t}+\Delta\right) P+2\langle\nabla P, \nabla f\rangle \\
& =\left(2 \Delta f+2 S_{g}-\frac{m}{2 \tau}\right)-\tau\left(4 S_{i j} \nabla_{i} \nabla_{j} f-4 \alpha \tau_{g} u\langle\nabla u, \nabla f\rangle+\Delta|\nabla f|^{2}\right. \\
& \left.-2\langle\nabla f, \nabla \Delta f\rangle-2 S_{i j} \nabla_{i} f \nabla_{j} f+2\left|S_{i j}\right|^{2}+2 \alpha\left|\tau_{g} u\right|^{2}\right) \\
& =\left(2 \Delta f+2 S_{g}-\frac{m}{2 \tau}\right)-\tau\left(4 S_{i j} \nabla_{i} \nabla_{j} f+2|\nabla \nabla f|^{2}+2\left|S_{i j}\right|^{2}\right) \\
& -2 \tau \alpha\left(\mid \tau_{g} u+\langle\nabla u, \nabla f\rangle^{2}-2 \tau_{g} u\langle\nabla u, \nabla f\rangle\right) \\
& =-2 \tau\left(2 S_{i j} \nabla_{i} \nabla_{j} f+|\nabla \nabla f|^{2}+\left|S_{i j}\right|^{2}-\frac{1}{\tau}\left(\Delta f+R-\frac{m}{4 \tau}\right)\right) \\
& -2 \tau \alpha\left(\left|\tau_{g} u-\langle\nabla u, \nabla f\rangle\right|^{2}\right) \\
& =-2 \tau\left(\left(S_{i j}+\nabla_{i} \nabla_{j} f\right)^{2}-\frac{1}{\tau}\left(\Delta f+R-\frac{m}{4 \tau}\right)\right)-2 \tau \alpha\left(\left|\tau_{g} u-\langle\nabla u, \nabla f\rangle\right|^{2}\right) \\
& =-2 \tau\left|S_{i j}+\nabla_{i} \nabla_{j} f-\frac{1}{2 \tau} g_{i j}\right|^{2}-2 \tau \alpha\left(\left|\tau_{g} u-\langle\nabla u, \nabla f\rangle\right|^{2}\right),
\end{aligned}
$$

where we have used the following calculation by Bochner's identity:

$$
\begin{aligned}
\Delta|\nabla f|^{2}-2\langle\nabla f, \nabla \Delta f\rangle-2 S_{i j} \nabla_{i} f \nabla_{j} f & =2|\nabla \nabla f|^{2}+2\left(R_{i j}-S_{i j}\right) \nabla_{i} f \nabla_{j} f \\
& =2|\nabla \nabla f|^{2}+2 \alpha\langle\nabla u, \nabla f\rangle^{2} .
\end{aligned}
$$

Hence,

$$
H^{-1} \square^{*} V=-2 \tau\left|S_{i j}+\nabla_{i} \nabla_{j} f-\frac{1}{2 \tau} g_{i j}\right|^{2}-2 \tau \alpha\left(\left|\tau_{g} u-\langle\nabla u, \nabla f\rangle\right|^{2}\right)
$$

and

$$
\square^{*} V=-2 \tau\left|S_{i j}+\nabla_{i} \nabla_{j} f-\frac{1}{2 \tau} g_{i j}\right|^{2} H-2 \tau \alpha\left(\left|\tau_{g} u-\langle\nabla u, \nabla f\rangle\right|^{2}\right) H .
$$

The consequence of which is a localised version of Perelman's $\mathcal{W}$-entropy monotonicity formula. Thus, 


$$
\begin{aligned}
\frac{d \mathcal{W}}{d t}=\frac{\partial}{\partial t} \int_{M} V d \mu_{g} & =\int_{M}\left(\partial_{t} V-R V+\alpha|\nabla u|_{g}^{2} V\right) d \mu \\
& =\int_{M}\left(-\square^{*} V-\Delta_{g} V\right) d \mu_{g}=\int_{M}-\square^{*} V d \mu_{g} \\
= & 2(T-t) \int_{M}\left(\left|S_{i j}+\nabla_{i} \nabla_{j} f-\frac{1}{2(T-t)} g_{i j}\right|^{2}\right. \\
& \left.\quad+\alpha\left(\left|\tau_{g} u-\langle\nabla u, \nabla f\rangle\right|^{2}\right)\right) \frac{e^{-f}}{(4 \pi \tau)^{-m / 2}} d \mu_{g} .
\end{aligned}
$$

Proposition 3.7. With the assumptions of Theorem 3.2, we have

$$
\frac{\partial}{\partial t}\left(\int_{M} f H d \mu_{g}\right) \geq 0
$$

Proof. By direct computation,

$$
\begin{aligned}
\frac{\partial}{\partial t}\left(\int_{M} f H d \mu\right)= & \int_{M}\left(\frac{\partial}{\partial t} f H+f \frac{\partial}{\partial t} H-S_{g} f H\right) d \mu_{g} \\
= & \int_{M}\left(-\Delta_{g} f+|\nabla f|_{g}^{2}-S_{g}+\frac{m}{2 \tau}\right) H d \mu_{g} \\
& \quad+\int_{M} f\left(-\Delta_{g} H+S_{g} H\right) d \mu-\int_{M} S_{g} f H d \mu_{g} \\
= & \int_{M}\left(-2 \Delta_{g} f+|\nabla f|_{g}^{2}\right) H d \mu+\int_{M}\left(\frac{m}{2 \tau}-S_{g}\right) H d \mu_{g},
\end{aligned}
$$

where we used integration by parts on $-\int_{M} \Delta_{g} f H=-\int_{M} f \Delta_{g} H$. Rearranging the above, we have

$$
\begin{aligned}
\frac{\partial}{\partial t}\left(\int_{M} f H d \mu_{g}\right) & =\int_{M}\left(-S_{g}-2 \Delta_{g} f+|\nabla f|_{g}^{2}\right) H d \mu_{g}+\frac{m}{2 \tau} \int_{M} H d \mu_{g} \\
& =-\int_{M}\left(S_{g}+|\nabla f|_{g}^{2}\right) H d \mu_{g}+\frac{m}{2 \tau} \\
& =-\mathcal{F}_{\alpha}+\frac{m}{2 \tau},
\end{aligned}
$$

where $\mathcal{F}_{\alpha}=\int_{M}\left(S_{g}+|\nabla f|_{g}^{2}\right) H d \mu_{g}$ is the Perelman energy functional introduced in [Müller 2012], which we discussed in Section 2. Next is to show that

$$
\frac{\partial}{\partial t}\left(\int_{M} f u d \mu\right)=-\mathcal{F}_{\alpha}+\frac{m}{2 \tau} \geq 0 .
$$

Recall the evolution of $\mathcal{F}_{\alpha}$ :

$$
\frac{d}{d t} \mathcal{F}_{\alpha}(g, f)=2 \int_{M}\left(|\mathrm{Rc}-\alpha \nabla u \otimes \nabla u+\nabla \nabla f|^{2}+\alpha\left|\tau_{g} u-\langle\nabla u, \nabla f\rangle\right|^{2}\right) H d \mu_{g} .
$$


Straightforward analysis, using an elementary inequality and the Cauchy-Schwarz inequality, gives

$$
|\mathrm{Rc}-\alpha \nabla u \otimes \nabla u+\nabla \nabla f|^{2} \geq \frac{1}{m}\left(R_{g}-\alpha|\nabla u|_{g}^{2}+\Delta_{g} f\right)^{2}
$$

so that

$$
\int\left(S_{g}+\Delta_{g} f\right) H d \mu_{g} \leq\left(\int\left(S_{g}+\Delta_{g} f\right)^{2} H d \mu_{g}\right)^{1 / 2}\left(\int H d \mu_{g}\right)^{1 / 2},
$$

which implies

$$
\left(\int_{M}\left(S_{g}+\Delta_{g} f\right) H d \mu\right)^{2} \leq \int_{M}\left(S_{g}+\Delta_{g} f\right)^{2} H d \mu_{g} .
$$

Hence by (3-16) and (3-17), we obtain

$$
\frac{d}{d t} \mathcal{F}_{\alpha} \geq \frac{2}{m} \int_{M}\left(S_{g}+\Delta_{g} f\right)^{2} H d \mu_{g}+\int_{M} 2 \alpha\left|\tau_{g} u-\langle\nabla u, \nabla f\rangle\right|^{2} H d \mu_{g} .
$$

We can then solve

$$
\frac{d}{d t} \mathcal{F}_{\alpha} \geq \frac{2}{m} \mathcal{F}_{\alpha}^{2}, \quad \mathcal{F}_{\alpha} \geq 0
$$

This implies

$$
\begin{aligned}
\frac{d \mathcal{F}_{\alpha}}{\mathcal{F}_{\alpha}^{2}} \geq \frac{2}{m} d t \Longrightarrow-\left.\frac{1}{\mathcal{F}_{\alpha}}\right|_{t} ^{T} \geq \frac{2}{m}(T-t) \Longrightarrow \frac{1}{\mathcal{F}_{\alpha}(t)}-\frac{1}{\mathcal{F}_{\alpha}(T)} \geq \frac{2}{m} \tau & \\
& \Rightarrow \frac{1}{\mathcal{F}_{\alpha}(t)} \geq \frac{2}{m} \tau+\frac{1}{\mathcal{F}_{\alpha}(T)} .
\end{aligned}
$$

From here we can conclude as follows:

(i) Suppose $\mathcal{F}_{\alpha}(T)>0$. Then

$$
\frac{1}{\mathcal{F}(t)} \geq \frac{2 \tau}{m} ; \quad \text { i.e, } \mathcal{F}_{\alpha}(t) \leq \frac{m}{2 \tau} .
$$

(ii) Suppose $\mathcal{F}_{\alpha}(T) \leq 0$. Then $\mathcal{F}_{\alpha}(t) \leq 0 \leq m /(2 \tau)$ for all $t \in[0, T)$, since we know that $d \mathcal{F}_{\alpha} / d t \geq 0$.

Hence

$$
\mathcal{F}_{\alpha}(t) \leq \frac{m}{2 \tau} \quad \text { for } t \in[0, T),
$$

which proves the claim (3-15).

\section{Differential Harnack estimates}

In this section we obtain Perelman's differential Harnack-type estimate which holds for the fundamental solution and, of course, all positive solutions to the conjugate heat equation coupled to the Ricci-harmonic map flow. There is an improvement over some known results as there is no explicit restriction on the curvature and no 
recourse to Perelman's reduced distance. In what follows, we want to show that the local entropy satisfies a pointwise differential inequality for the positive minimal solution. Define a differential Harnack quantity

$$
P_{\epsilon}:=\frac{\epsilon^{2} \tau}{4 \pi}\left(2 \Delta f-|\nabla f|^{2}+S_{g}\right)+f+\frac{m}{2} \ln \frac{4 \pi}{\epsilon^{2}}-\frac{m \epsilon^{2}}{4 \pi} .
$$

Theorem 4.1. Let $M$ be a closed manifold with bounded Ricci curvature and $H(x, y, t)=H=(4 \pi t)^{-n / 2} e^{-f}$ satisfy $\square^{*} H=0$, where $H$ tends to a $\delta$-function as $t \rightarrow T$ and satisfies $\int_{M} H d \mu_{g}=1$. Then for all $t<T$ and $\epsilon^{2} \rightarrow 4 \pi$ as $t \rightarrow T$, we have

$$
\frac{\epsilon^{2} \tau}{4 \pi}\left(2 \Delta f-|\nabla f|^{2}+S_{g}\right)+f-\frac{m \epsilon^{2}}{4 \pi} \leq 0 .
$$

Proof. Let $h$ be any compactly supported smooth function for all $t_{0}>0$. Suppose $h(\cdot, t)$ is a positive solution to the ordinary heat equation $\left(\partial_{t}-\Delta\right) h=0$ (this is Perelman's argument in [2002, Corollary 9.3]). Then, it is clear that

$$
\frac{\partial}{\partial t} \int_{M} H h d V=0
$$

and we have by direct calculation that

$$
\begin{aligned}
\frac{\partial}{\partial t} \int_{M} h P_{\epsilon} H d \mu_{g} & =\int_{M}\left(\partial_{t} h\left(P_{\epsilon} H\right)+h \partial_{t}\left(P_{\epsilon} H\right)-S_{g} P_{\epsilon} H\right) d \mu_{g} \\
& \left.=\int_{M}\left(\left(\partial_{t}-\Delta\right) h\left(P_{\epsilon} H\right)+h\left(\partial_{t}+\Delta-S_{g}\right) P_{\epsilon} H\right)\right) d \mu_{g} \\
& =-\int_{M} h \square^{*}\left(P_{\epsilon} H\right) d \mu_{g} \\
& =-\frac{\epsilon^{2}}{4 \pi} \int_{M} h \square^{*} V_{\epsilon} d \mu_{g} \geq 0 .
\end{aligned}
$$

The inequality is due to Proposition 3.6. We are left to show that for the everywhere positive function $h(\cdot, t)$, the limit of $\int_{M} h V_{\epsilon} d \mu_{g}$ is nonpositive as $t \rightarrow T$. We assume the claim a priori, i.e, $\lim _{t \rightarrow T} \int_{M} h V_{\epsilon} d \mu_{g}=0$, with

$$
V_{\epsilon}=\left(\tau\left(2 \Delta f-|\nabla f|^{2}+S_{g}\right)+\frac{4 \pi}{\epsilon^{2}} f-m\right) H,
$$

and conclude the result.

For completeness, we devote the next effort to justifying the claim

$$
\lim _{t \rightarrow T} \int_{M} h V_{\epsilon} d \mu_{g} \leq 0 \Longleftrightarrow \lim _{t \rightarrow T} \int_{M} h P_{\epsilon} H d \mu_{g} \leq \frac{m}{2} \lim _{t \rightarrow T}\left(\ln \frac{4 \pi}{\epsilon^{2}} \int_{M} h H d \mu_{g}\right) .
$$

Our argument follows from [Ni 2006; Perelman 2002] and can be compared with the recent preprint [Băileşteanu and Tran 2013, Proposition 4.2] (see also [Chow 
et al. 2008, Section 16.4]), where we know that $\lim _{t \rightarrow T} \int_{M} V h d \mu_{g} \leq 0$ (where $V$ is as defined in Proposition 3.6). To see this clearly, we write

$$
P_{\epsilon} H=\frac{\epsilon^{2}}{4 \pi} V_{\epsilon}+\frac{m}{2} \ln \frac{4 \pi}{\epsilon^{2}} H,
$$

which implies

$$
\lim _{t \rightarrow T} \int_{M} h P_{\epsilon} H d \mu_{g}=\frac{\epsilon^{2}}{4 \pi} \lim _{t \rightarrow T} \int_{M} V_{\epsilon} h d \mu_{g}+\frac{m}{2} \ln \frac{4 \pi}{\epsilon^{2}} \lim _{t \rightarrow T} \int_{M} h H d \mu_{g} .
$$

If $H$ tends to a Dirac $\delta$-function, say at a point $p \in M$, for $\tau \rightarrow T$, then $f$ satisfies $f(x, \tau) \rightarrow d^{2}(p, x) / 4 \tau$. This is in relation to the $l$-length of Perelman. This yields

$$
\lim _{\tau \rightarrow 0} \int_{M} f h H d \mu_{g} \leq \limsup _{\tau \rightarrow 0} \int_{M} \frac{d^{2}(p, x)}{4 \tau} h H d \mu_{g}=\frac{m}{2} h(p, T) .
$$

Meanwhile, by the strong maximum principle, we have $h(x, T)>0$ and

$$
\lim _{\tau \rightarrow 0} \int_{M} h H d \mu_{g}=h(x, T) .
$$

Hence by a scaling argument, we assume that $h(x, T)=1$. Rewriting $P_{\epsilon}$ and using integration by parts, we have

$$
\begin{aligned}
\int_{M} P_{\epsilon} h H d \mu_{g}=\int_{M} \frac{\epsilon^{2} \tau}{4 \pi}\left(|\nabla f|^{2}\right. & \left.+S_{g} d \mu_{g}-\frac{m}{2 \tau}\right) h H d \mu_{g}-\frac{\epsilon^{2} \tau}{2 \pi} \int_{M}\langle\nabla f, \nabla h\rangle H d V \\
& +\int_{M} f H h d \mu_{g}+\frac{m}{2}\left(\ln \frac{4 \pi}{\epsilon^{2}}-\frac{\epsilon^{2}}{4 \pi}\right) \int_{M} H h d \mu_{g} .
\end{aligned}
$$

We should also note that since $h\left(\cdot, t_{0}\right)$ is compactly supported and by the strong maximum principle, we have that $h\left(\cdot, t_{0}\right),\left|\nabla h\left(\cdot, t_{0}\right)\right|$ and $\left|\Delta h\left(\cdot, t_{0}\right)\right|$ are bounded on $M$. This implies that there exists a bounded solution $h\left(\cdot, t_{0}\right)$. Now we claim that the first three terms on the right-hand side of the last equation vanish as $\tau \rightarrow 0$. We can see this, for instance, in the following argument: By integration by parts and the fact that $\nabla H=-H \nabla f$, we have

$$
-\tau \int_{M}\langle\nabla f, \nabla h\rangle H \mu_{g}=\tau \int_{M}\langle\nabla H, \nabla h\rangle \mu_{g}=-\tau \int_{M} H \Delta h \mu_{g}
$$

is bounded since $|\Delta h|$ is bounded as stated earlier. Thus, the second term in righthand side of the preceding equation is bounded and goes to zero as $\tau \rightarrow 0$, so the same is true for first terms (which follows from gradient estimates [Chow et al. 2008, Lemma 16.47]). Thus the analysis is reduced to showing that

$$
\lim _{\tau \rightarrow 0} \int V_{\epsilon} h d \mu_{g}<C(m) \leq 0 .
$$


By the monotonicity formula for $W_{\alpha, \epsilon}$, we have

$$
\frac{\partial}{\partial t} \int_{M} P_{\epsilon} h H d \mu_{g}=\frac{\epsilon^{2} \tau}{4 \pi} \frac{\partial}{\partial t} \int_{M} V_{\epsilon} h d \mu_{g} \geq 0 .
$$

By the mean value theorem, there exists a sequence $\tau_{k} \rightarrow 0$ such that $\lim _{\tau_{k} \rightarrow 0} \tau_{k} \int_{M}\left(\left|\operatorname{Rc}-\alpha \nabla u \otimes \nabla u+\nabla \nabla f-\frac{n}{2 \tau_{k}} g\right|^{2}+\alpha\left|\tau_{g} u-\langle\nabla u, \nabla f\rangle\right|^{2}\right) H h d \mu_{g}=0$. Applying the Cauchy-Schwarz and Hölder inequalities, we have

$$
\left|\mathrm{Rc}-\alpha \nabla u \otimes \nabla u+\nabla \nabla f-\frac{1}{2 \tau_{k}} g\right|^{2} \geq \frac{1}{m}\left(R_{g}-\alpha|\nabla u|_{g}^{2}+\Delta_{g} f-\frac{n}{2 \tau_{k}} g\right)^{2}
$$

and

$$
\begin{aligned}
& \int_{M} \tau_{k}\left(S_{g}+\Delta f-\frac{n}{2 \tau_{k}}\right) H h d \mu_{g} \\
& \leq\left(\tau_{k}^{2} \int_{M}\left(S_{g}+\Delta f-\frac{n}{2 \tau_{k}}\right)^{2} H h d \mu_{g}\right)^{1 / 2}\left(\int_{M} H h d \mu_{g}\right)^{1 / 2} \\
& \leq \sqrt{m}\left(\tau_{k}^{2} \int_{M}\left|\operatorname{Rc}-\alpha \nabla u \otimes \nabla u+\nabla \nabla f-\frac{1}{2 \tau_{k}}\right|^{2} H h d \mu_{g\left(\tau_{k}\right)}\right)^{1 / 2}\left(\int_{M} H h d \mu_{g}\right)^{1 / 2} \rightarrow 0
\end{aligned}
$$

as $\tau_{k} \rightarrow 0$, since $\alpha\left|\tau_{g} u-\langle\nabla u, \nabla f\rangle\right|^{2} \geq 0$ and $\lim _{\tau_{k} \rightarrow 0} \int_{M} H h d \mu_{g\left(\tau_{k}\right)}$ is finite.

Then we have

$$
\lim _{t \rightarrow T} \int V_{\epsilon} h d \mu_{g}
$$

$$
\begin{aligned}
= & \lim _{t \rightarrow T} \int_{M}\left(\frac{\epsilon^{2}}{4 \pi} \tau_{k}\left(2 \Delta f-|\nabla f|^{2}+S_{g}\right)+\frac{4 \pi}{\epsilon^{2} \tau} f-m\right) H h d \mu_{g} \\
= & \lim _{t \rightarrow T} \int_{M}\left(\frac{\epsilon^{2} \tau_{k}}{4 \pi}\left(\Delta f+S_{g}-\frac{n}{2 \tau_{k}}\right)\right) H h d \mu_{g\left(\tau_{k}\right)} \\
& +\lim _{t \rightarrow T} \int_{M}\left(\frac{\epsilon^{2} \tau_{k}}{4 \pi}\left(\Delta f-|\nabla f|^{2}\right)\right) H h d \mu_{g}+\lim _{t \rightarrow T} \int_{M}\left(f-\frac{m \epsilon^{2}}{8 \pi}\right) H h d \mu_{g} \\
= & \lim _{t \rightarrow T} \int_{M}\left(f-\frac{m \epsilon^{2}}{8 \pi}\right) H h d \mu_{g},
\end{aligned}
$$

where we have used the identity

$$
\int_{M}\left(\Delta f-|\nabla f|^{2}\right) H d \mu=-\int_{M} \Delta H d \mu=0
$$

for any positive solution $H$ and the fact that each quantity in (4-5) is bounded to obtain $\lim _{t \rightarrow T} \tau_{k} \int_{M}\left(\Delta f-|\nabla f|^{2}\right) H h d \mu_{g}=0$. 
By (4-4) and the asymptotic behaviour of the heat kernel, i.e, $f \approx d^{2} /(4 \tau)$ as $\tau \rightarrow 0$, we have (see [Ni 2006, Theorem 2.1])

$$
H(x, y, \tau) \sim(4 \pi \tau)^{-m / 2} \exp \left(\frac{d^{2}(x, y)}{4 \tau}\right) \sum_{j=0}^{\infty} u_{j}(x, y, \tau) \tau^{j}:=w_{k}(x, y, \tau)
$$

as $\tau \rightarrow 0$, where $d^{2}(x, y)$ is the distance function and $w_{k}(x, y, t)$ satisfies

$$
w_{k}(x, y, \tau)=O\left(\tau^{k+1-m / 2} \exp \left(\frac{\delta d^{2}(x, y)}{4 \tau}\right)\right)
$$

uniformly for all $x, y \in M$ and $\delta$ is just a number depending only on the geometry of $(M, g)$. The function can be chosen such that $u_{0}(x, y, 0)=1$. Though, the above asymptotic result does not require any curvature assumption, a result due to Cheeger and Yau [1981] states that on a manifold with bounded Ricci curvature (which is our case), the heat kernel satisfies

which implies

$$
H(x, y, \tau) \geq(4 \pi \tau)^{-m / 2} \exp \left(\frac{d^{2}(x, y)}{4 \tau}\right),
$$

Therefore,

$$
f(x, \tau) \leq \frac{d^{2}(x, y)}{4 \tau}
$$

$$
\begin{aligned}
\lim _{\tau \rightarrow 0} \int_{M}\left(f-\frac{m \epsilon^{2}}{8 \pi}\right) h H d \mu_{g} & \leq \limsup _{\tau \rightarrow 0} \int_{M}\left(\frac{d^{2}(x, y)}{4 \tau}-\frac{m \epsilon^{2}}{8 \pi}\right) h(y, t) H(x, y, \tau) d \mu_{g} \\
& =\limsup _{\tau \rightarrow 0} \int_{M}\left(\frac{d^{2}(x, y)}{4 \tau}-\frac{m \epsilon^{2}}{8 \pi}\right) \frac{e^{-d^{2}(x, y) / 4 \tau}}{(4 \pi \tau)^{m / 2}} h(y, t) d \mu_{g} .
\end{aligned}
$$

It is easy to see that for any $\delta>0$, the integration of the above integrand in the domain $d(x, y) \geq \delta$ converges to zero. Therefore,

$$
\begin{aligned}
\lim _{t \rightarrow 0} \int_{M}\left(f-\frac{m \epsilon^{2}}{8 \pi}\right) h H d \mu_{g} & \\
& \leq \lim _{t \rightarrow 0} \int_{d(x, y) \leq \delta}\left(\frac{d^{2}(x, y)}{4 t}-\frac{m \epsilon^{2}}{8 \pi}\right) \frac{e^{-d^{2}(x, y) /(4 t)}}{(4 \pi t)^{m / 2}} h(y, t) d \mu_{g} .
\end{aligned}
$$

Whenever $\delta$ is chosen sufficiently small, $d(x, y)$ is asymptotically sufficiently close to the Euclidean distance. Then by a standard approximation using local coordinates, we have

$$
\begin{aligned}
\lim _{t \rightarrow 0} \int_{M}\left(\frac{d^{2}(x, y)}{4 t}-\frac{m \epsilon^{2}}{8 \pi}\right) h H d \mu_{g} \\
\quad=\lim _{t \rightarrow 0} \int_{\mathbb{R}^{m}}\left(\frac{|x-y|^{2}}{4 \tau}-\frac{m \epsilon^{2}}{8 \pi}\right) \frac{e^{-|x-y|^{2} /(4 \tau)}}{(4 \pi \tau)^{m / 2}} h_{p}(y) d y,
\end{aligned}
$$


where $h_{p}$ is the pullback of $h(\cdot, 0)$ from the region $d(x, y) \leq \delta$ to the Euclidean space.

Splitting the last integrand as in [Kuang and Zhang 2008], we are left with

$$
\begin{aligned}
\lim _{t \rightarrow 0} \int_{M}\left(f-\frac{m \epsilon^{2}}{8 \pi}\right) h H d \mu_{g} & \leq h_{p}(x) \lim _{t \rightarrow 0} \int_{\mathbb{R}^{m}}\left(\frac{|x-y|^{2}}{4 \tau}-\frac{m \epsilon^{2}}{8 \pi}\right) \frac{e^{-|x-y|^{2} /(4 t)}}{(4 \pi \tau)^{m / 2}} d y \\
& =h_{p}(\cdot) \lim _{\tau \rightarrow 0} \int_{\mathbb{R}^{m}}\left(\frac{|y|^{2}}{4 \tau} \frac{e^{-|y|^{2} /(4 \tau)}}{(4 \pi \tau)^{m / 2}}\right) d y-\frac{m \epsilon^{2}}{8 \pi} h_{p}(\cdot) .
\end{aligned}
$$

Lastly, we have that the right-hand side evaluates to a constant $C(m) \leq 0$ by using the standard Gauss integral

$$
\int_{\mathbb{R}^{m}}\left(\frac{|y|^{2}}{4 \tau} \frac{e^{-|y|^{2} /(4 \tau)}}{(4 \pi \tau)^{n / 2}}\right) d y=\frac{m}{2}
$$

and the condition $\epsilon \rightarrow 2 \sqrt{\pi}$ as $\tau \rightarrow 0$. The claim then follows.

Finally in this section we prove Perelman's differential Harnack estimates for $f$ as an application of Theorem 4.1 and the monotonicity of $\mathcal{W}_{\epsilon, \alpha}$. A corollary to this gives estimates of Li-Yau type for all positive solutions $H(x, \tau)$.

Proposition 4.2. Let the assumptions of Theorem 4.1 hold. Then for any smooth curve $\gamma(\tau)$ in $M$, we have the estimate

$$
-\frac{d}{d t} f(\gamma(\tau), \tau) \leq \frac{1}{2}\left(\left|\gamma^{\prime}(\tau)\right|^{2}+S_{g}(\gamma(\tau), \tau)-\frac{m}{2 \tau}\right) .
$$

After the usual integration of (4-9) along the path $\gamma(\tau)$ and exponentiation, we have the following result.

Corollary 4.3. With the notation and assumptions of Theorem 4.1, the following Li-Yau Harnack estimate holds:

$$
\frac{H\left(x_{2}, t_{2}\right)}{H\left(x_{1}, t_{1}\right)} \leq\left(\frac{T-t_{1}}{T-t_{2}}\right)^{m / 4} \exp \left(\frac{1}{2} \int_{t_{1}}^{t_{2}}\left(\left|\gamma^{\prime}(t)\right|^{2}+S_{g}(\gamma(t), t) d t\right) .\right.
$$

Proof of Proposition 4.2. Precisely from (4-1), we have

$$
f \leq \frac{m \epsilon^{2}}{4 \pi}-\frac{\epsilon^{2} \tau}{4 \pi}\left(2 \Delta f-|\nabla f|^{2}+S_{g}\right) \leq \frac{m \epsilon^{2}}{8 \pi}
$$

since $\Delta f+S_{g}-m /(2 \tau) \geq 0$ by the monotonicity formula (3-3). Now multiplying (4-1) through by $2 \pi /\left(\epsilon^{2} \tau\right)$, we have

$$
\Delta f-\frac{1}{2}|\nabla f|^{2}+\frac{1}{2} S_{g}+\frac{2 \pi}{\epsilon^{2} t} f-\frac{m}{2 \tau} \leq 0 .
$$

Using $\Delta f=-\partial_{t} f+|\nabla f|^{2}-S_{g}+m /(2 \tau)$ from (2-9), we obtain

$$
-\partial_{t} f+\frac{1}{2}|\nabla f|^{2} \leq \frac{1}{2} S_{g}-\frac{2 \pi}{\epsilon^{2} t} f .
$$


By Young's inequality, we have

$$
\begin{aligned}
-\frac{d}{d t} f(\gamma(\tau), \tau) & =-\partial_{t} f(\gamma(\tau), \tau)-\left\langle\nabla f(\gamma(\tau), \tau), \gamma^{\prime}(\tau)\right\rangle \\
& \leq-\partial_{t} f+\frac{1}{2}|\nabla f|^{2}+\frac{1}{2}\left|\gamma^{\prime}(\tau)\right|^{2} \\
& =\frac{1}{2}\left|\gamma^{\prime}(\tau)\right|^{2}+\frac{1}{2} S_{g}(\gamma(\tau), \tau)-\frac{2 \pi}{\epsilon^{2} \tau} f(\gamma(\tau) \tau)
\end{aligned}
$$

on the path $\gamma(\tau)$. The result follows by using the fact that $f \leq m \epsilon^{2} /(8 \pi)$.

\section{Log-Sobolev inequalities along $(\mathrm{RH})_{\alpha}$-flow}

By the results of Aubin [1976] and Hebey [1996] for complete manifolds whose Ricci curvature is bounded from below and injectivity radius is positive and bounded from above, we can assume the Sobolev embedding on the initial metric, since $(M, g(0))$ is a compact Riemannian manifold. Let $A_{0}, B_{0}<\infty$ be positive constants such that for all $v \in W^{1,2}\left(M, g_{0}\right)$,

$$
\|v\|_{2 m /(m-2)} \leq A_{0}\|\nabla v\|_{2}+B_{0}\|v\|_{2},
$$

where $A_{0}$ and $B_{0}$ depend only on $m, g_{0}$, the lower bound for the Ricci curvature and the injectivity radius. We can then write (5-1) as

$$
\left(\int_{M} v^{2 m /(m-2)} d \mu_{g_{0}}\right)^{(m-2) / m} \leq A \int_{M}\left(4|\nabla v|^{2}+S_{g} v^{2}\right) d \mu_{g_{0}}+B \int_{M} v^{2} d \mu_{g_{0}},
$$

where

$$
A=\frac{1}{4} A_{0} \quad \text { and } \quad B=\frac{1}{4} A_{0} \sup S_{g}^{-}(\cdot, 0)+B_{0}
$$

since $S_{g}(x, 0)+\sup S_{g}^{-}(\cdot, 0)=S_{g}^{+}(x, 0)-S_{g}^{-}(x, 0)$. We will assume that (5-2) holds uniformly for $g(t), t>0$, and different $A$ and $B$ in order to prove the logarithmic Sobolev inequalities.

The usual way of deriving logarithmic Sobolev inequalities follows from a careful application of Hölder's and Jensen's inequalities since $\log v$ is a concave function, in which case

$$
\int v^{2} \ln v^{q-2} d \mu \leq \ln \int v^{q} d \mu
$$

with the assumption that $\int v^{2} d \mu=1$. Then

$$
\int v^{2} \ln v d \mu \leq \frac{q}{q-2} \ln \left(\int v^{q} d \mu\right)^{1 / q} .
$$

Taking $q=2 m /(m-2)$, we have

$$
\int v^{2} \ln v d \mu \leq \frac{m}{2} \ln \left(\int v^{2 m /(m-2)} d \mu\right)^{(m-2) / 2 m},
$$


and by multiplying both sides by 2 we obtain the following result.

Lemma 5.1. For any $v \in W^{1,2}\left(M, g_{0}\right)$ with $\|v\|_{2}=1$,

$$
\int_{M} v^{2} \ln v^{2} d \mu_{g_{0}} \leq \frac{m}{2} \ln \left(A \int_{M}\left(4|\nabla v|^{2}+S_{g} v^{2}\right) d \mu_{g_{0}}+B\right) .
$$

See [Hsu 2008; Ye 2007; Zhang 2007] for similar proofs. Inequalities of the form (5-3) are usually estimated further by the application of an elementary inequality of the form $\ln y \leq \theta y-\ln \theta-1$, where $\theta, y \geq 0$. Precisely, taking

$$
y=A \int_{M}\left(4|\nabla v|^{2}+S_{g} v^{2}\right) d \mu_{g_{0}}+B
$$

in (5-3) gives us

$$
\begin{aligned}
\int_{M} v^{2} \ln v^{2} d \mu_{g_{0}} & \leq \frac{m \theta}{2}\left(A \int_{M}\left(4|\nabla v|^{2}+S_{g} v^{2}\right) d \mu_{g_{0}}+B\right)-\frac{m}{2}(1+\ln \alpha) \\
& =\frac{m \theta A}{2} \int_{M}\left(4|\nabla v|^{2}+S_{g} v^{2}\right) d \mu_{g_{0}}+\frac{m \theta B}{2}-\frac{m}{2}-\frac{m}{2} \ln \alpha .
\end{aligned}
$$

We will now modify the arguments in both [Ye 2007] and [Zhang 2007] to prove the following result which says the monotonicity of the $\mathcal{W}_{\alpha, \epsilon}$-entropy implies a logarithmic Sobolev inequality (not with the best constant). Here we assume the flow exists for all time.

Theorem 5.2. Let $(M, g)$ be a compact Riemannian manifold of dimension $m \geq 3$ and the metric $g(t)$ evolved by the $(\mathrm{RH})_{\alpha}$-flow. Assume that an $L^{2}$-Sobolev embedding (5-2) holds true with respect to the initial metric $g(0)=g_{0}$. Then, we have

$\int_{M} v^{2} \ln v^{2} d \mu_{g(t)} \leq \int_{M} \sigma^{2}\left(4|\nabla v|^{2}+S_{g} v^{2}\right) d \mu_{g(t)}-\frac{m}{2} \ln \sigma^{2}+\left(t+\sigma^{2}\right) \beta_{1}+\frac{m}{2} \ln \frac{m A}{2 e}$, where $\sigma>0, \beta_{1}=4 A_{0}^{-1} B_{0}+\sup S_{g}^{-}(\cdot, 0)$ and

$$
\lambda_{\alpha 0}=\inf _{\|v\|_{2}=1} \int_{M}\left(4|\nabla v|^{2}+S_{g} v^{2}\right) d \mu_{g_{0}} ;
$$

that is, $\lambda_{\alpha 0}$ is the first eigenvalue of the operator $-4 \Delta+S_{g}$.

Moreover, if $\lambda_{\alpha 0}$ is strictly positive for $S_{g}(\cdot, 0)>0\left(\right.$ i.e., $\left.R(\cdot, 0)>\alpha(0)|\nabla u(0)|^{2}\right)$, then

(5-6)

$\int_{M} v^{2} \ln v^{2} d \mu_{g(t)} \leq \int_{M} \sigma^{2}\left(4|\nabla v|^{2}+S_{g} v^{2}\right) d \mu_{g(t)}-\frac{m}{2} \ln \sigma^{2}+\left(t+\sigma^{2}\right) \beta_{2}+\frac{m}{2} \ln \frac{m A}{2 e}$ holds with $B_{0}=0$, i.e., $\beta_{2}=\sup S_{g}^{-}(\cdot, 0)$. 
We first discuss some vital issues that will help put the proof of the above theorem in perspective. Now take an $L^{2}$-solution $H=H(x, t)$ of the conjugate heat equation

$$
\partial_{t} H=-\Delta H+S_{g} H
$$

to be $H=(4 \pi \tau)^{m / 2} e^{-f}$. Relating the entropy $\mathcal{W}_{\alpha, \epsilon}$ with the idea of logarithmic Sobolev inequalities, we consider a function

$$
v=\sqrt{H}=\frac{e^{-f / 2}}{(4 \pi \tau)^{m / 4}}
$$

such that $\int_{M} v^{2} d \mu=1$. We also notice that (5-8) implies $f=-\ln v^{2}-(m / 2) \ln \tau-$ $(m / 2) \ln (4 \pi)$; hence the entropy (3-1) is rewritten as

$$
\begin{aligned}
\mathcal{W}_{\epsilon}(g, v, \tau)= & \frac{\epsilon^{2}}{4 \pi} \int_{M}\left(\tau\left(4|\nabla v|^{2}+S_{g} v^{2}\right)-v^{2} \ln v^{2}\right) d \mu-\frac{\epsilon^{2}}{4 \pi} \frac{m}{2} \ln \tau \\
& -\frac{\epsilon^{2}}{4 \pi} \frac{m}{2} \ln (4 \pi)+\left(1-\frac{\epsilon^{2}}{4 \pi}\right) \int_{M} f v^{2} d \mu-\frac{m \epsilon^{2}}{4 \pi}+\frac{m}{2} \ln \frac{4 \pi}{\epsilon^{2}} .
\end{aligned}
$$

Define

$$
\mathcal{W}_{\epsilon}^{*}(g, v, \tau)=\frac{\epsilon^{2}}{4 \pi} \int_{M}\left(\tau\left(4|\nabla v|^{2}+S_{g} v^{2}\right)-v^{2} \ln v^{2}\right) d \mu
$$

and

$$
\mu_{\epsilon}^{*}(g, v, \tau)=\inf \left\{\mathcal{W}_{\epsilon}^{*}(g, v, \tau): \int_{M} v^{2} d \mu=1\right\} .
$$

Set $T^{*}=t^{*}+\sigma^{2}$ and $\tau(t)=T^{*}-t$ for $0 \leq t \leq t^{*}, \sigma>0$. Then

$$
\begin{aligned}
& \frac{d}{d t} \mathcal{W}_{\epsilon}(g, v, \tau) \\
& \quad=\frac{d}{d t} \mathcal{W}_{\epsilon}^{*}(g, v, \tau)-\frac{m \epsilon^{2}}{8 \pi} \frac{d}{d t} \ln \tau+\left(1-\frac{\epsilon^{2}}{4 \pi}\right) \frac{\partial}{\partial t} \int_{M} f v^{2} d \mu+\frac{m}{2} \ln \frac{4 \pi}{\epsilon^{2}} \geq 0,
\end{aligned}
$$

where the last inequality is due to the monotonicity of $\mathcal{W}_{\epsilon}(g, f, \tau)$, the proof of which also reveals that

$$
\frac{\partial}{\partial t} \int_{M} f v^{2} d \mu=-\mathcal{F}_{\alpha}+\frac{m}{2 \tau},
$$

where $\mathcal{F}_{\alpha}=\int_{M}\left(S_{g}+|\nabla f|^{2}\right) v^{2} d \mu$ is Perelman and Müller's energy functional. Let $\lambda_{\alpha 0}$ be the first eigenvalue of the operator $-4 \Delta+S_{g}$. Then, we know that $\lambda_{\alpha 0}=\inf _{\|u\|_{2}=1} \mathcal{F}_{\alpha}$. Therefore we arrive at

$$
\frac{d}{d t} \mathcal{W}_{\epsilon}^{*} \geq \frac{n \epsilon^{2}}{8 \pi} \frac{d}{d t} \ln \tau+\left(1-\frac{\epsilon^{2}}{4 \pi}\right) \lambda_{\alpha 0} .
$$


To continue this argument, we should note that either (5-7) or (5-8) implies that the function $f=f(t)$ satisfies the following backward heat equation

$$
\frac{\partial f}{\partial t}=-\Delta f+|\nabla f|^{2}-S_{g}+\frac{m}{2 \tau},
$$

with $v=v(x, t)$ satisfying

$$
\frac{\partial v}{\partial t}=-\Delta v+\frac{|\nabla v|^{2}}{v}+\frac{S_{g}}{2} v
$$

on $\left[0, t^{*}\right]$ with a given terminal value at $t+t^{*}$ with $g=g\left(t^{*}\right)$.

Let $v_{0}$ be a minimizer of the entropy $\mathcal{W}_{\epsilon}\left(g, f, \tau_{0}\right)$ for all $v$ with $\int_{M} v_{0}^{2} d \mu_{g\left(t_{0}\right)}=1$. We can then solve heat equation (5-12) backward in time with initial data $f\left(t_{0}\right)=f_{0}$ and $v_{0}$ chosen at $t=t_{0}$. Let $u_{j}$ be the value of the conjugate heat equation (5-13) at $t=t_{j}$. We can define functions $f_{j}, j=1,2$, by

$$
u_{j}=\frac{e^{-f_{j} / 2}}{\left(4 \pi \tau_{j}\right)^{n / 4}}, \quad j=1,2 .
$$

Then by the monotonicity of $\mathcal{W}_{\alpha \epsilon}(g, f, \tau)$-entropy, using Perelman's approach we have

$$
\begin{aligned}
\mu_{\epsilon}\left(g\left(t_{1}\right), \tau\left(t_{1}\right)\right) & =\inf _{\left\|v_{0}\right\|_{g\left(t_{1}\right)}=1} \mathcal{W}_{\epsilon}\left(g\left(t_{1}\right), f_{0}, \tau_{1}\right) \leq \mathcal{W}_{\epsilon}\left(g\left(t_{1}\right), f_{1}, \tau_{1}\right) \\
& \leq \mathcal{W}_{\epsilon}\left(g\left(t_{2}\right), f_{2}, \tau_{2}\right)=\inf _{\left\|v_{0}\right\|_{g\left(t_{2}\right)}=1} \mathcal{W}_{\epsilon}\left(g\left(t_{2}\right), f, \tau_{2}\right)=\mu_{\epsilon}\left(g\left(t_{2}\right), \tau\left(t_{2}\right)\right) .
\end{aligned}
$$

It follows from the above that

$$
\mu_{\epsilon}^{*}\left(g\left(t_{1}\right), \tau\left(t_{1}\right)\right) \leq \mu_{\epsilon}^{*}\left(g\left(t_{2}\right), \tau\left(t_{2}\right)\right)+\frac{n \epsilon^{2}}{8 \pi} \ln \frac{\tau_{1}}{\tau_{2}}
$$

for any $t_{1}<t_{2}$, where $\tau_{j}=\tau\left(t_{j}\right), j=1,2$. Choosing $t_{1}=0$ and $t_{2}=t^{*}$, we then obtain

$$
\mu_{\epsilon}^{*}\left(g(0), t^{*}+\sigma^{2}\right) \leq \mu_{\epsilon}^{*}\left(g\left(t^{*}\right), \sigma^{2}\right)+\frac{n \epsilon^{2}}{8 \pi} \ln \frac{t^{*}+\sigma^{2}}{\sigma} .
$$

Since $0<t^{*}<T$ is arbitrary, we can write (5-14) as

$$
\mu_{\epsilon}^{*}\left(g(t), \sigma^{2}\right) \geq \mu_{\epsilon}^{*}\left(g(0), t+\sigma^{2}\right)+\frac{n \epsilon^{2}}{8 \pi} \ln \frac{\sigma^{2}}{t+\sigma^{2}}
$$

for all $t \in[0, T) .{ }^{1}$ We now state the proof.

Proof. We now apply (5-4) with $g=g_{0}$ to estimate $\mu_{\epsilon}^{*}\left(g(0), t+\sigma^{2}\right)$. For any function $v \in W^{1,2}(M, g)$ with $\|v\|_{2}=1$ and using

$$
\frac{m \theta A}{2}=t+\sigma^{2} \Rightarrow \theta=\frac{8\left(t+\sigma^{2}\right)}{n A_{0}},
$$

\footnotetext{
${ }^{1}$ The case $t=0$ is optimal, as equality is attained.
} 
the inequality in (5-4) becomes

$$
\begin{aligned}
& \int_{M} v^{2} \ln v^{2} d \mu_{g_{0}} \leq\left(t+\sigma^{2}\right) \int_{M}\left(4|\nabla v|^{2}+S_{g} v^{2}\right) d \mu_{g_{0}}+\frac{m}{2} \frac{8\left(t+\sigma^{2}\right)}{m A_{0}} B \\
&-\frac{m}{2} \ln \frac{8\left(t+\sigma^{2}\right)}{n A_{0}}-\frac{m}{2} \\
&=\left(t+\sigma^{2}\right) \int_{M}\left(4|\nabla v|^{2}+S_{g} v^{2}\right) d \mu_{g_{0}}+4\left(t+\sigma^{2}\right) B A_{0}^{-1} \\
&-\frac{m}{2} \ln \left(t+\sigma^{2}\right)+\frac{m}{2}\left(\ln A_{0}+\ln m-3 \ln 2-1\right) .
\end{aligned}
$$

Choosing $\epsilon^{2} \leq 4 \pi$ as before, it then follows that

$$
\begin{aligned}
& \mu_{\epsilon}^{*}\left(g(0), t+\sigma^{2}\right) \\
& \geq \frac{m \epsilon^{2}}{4 \pi}\left(\frac{1}{2} \ln \left(t+\sigma^{2}\right)-\frac{4}{m}(t+\sigma) B A_{0}^{-1}-\frac{1}{2}\left(\ln A_{0}+\ln m-3 \ln 2-1\right)\right) .
\end{aligned}
$$

Combining (5-15) and (5-16), we obtain

$\mu_{\epsilon}^{*}\left(g(t), \sigma^{2}\right) \geq \frac{m \epsilon^{2}}{8 \pi} \ln \sigma^{2}-\frac{m \epsilon^{2}}{\pi}\left(t+\sigma^{2}\right) B A_{0}^{-1}-\frac{m \epsilon^{2}}{8 \pi}\left(\ln A_{0}+\ln m-3 \ln 2-1\right)$,

which implies

$$
\begin{aligned}
\frac{\epsilon^{2}}{4 \pi} \int_{M}\left(\sigma^{2}\left(4|\nabla v|^{2}+S_{g} v^{2}\right)-\right. & \left.v^{2} \ln v^{2}\right) d \mu \\
& \geq \frac{m \epsilon^{2}}{8 \pi} \ln \sigma^{2}-\frac{m \epsilon^{2}}{\pi}\left(t+\sigma^{2}\right) B A_{0}^{-1}-\frac{m \epsilon^{2}}{8 \pi} \ln \frac{m A_{0}}{8 e} .
\end{aligned}
$$

Therefore,

$\int_{M} v^{2} \ln v^{2} d \mu \leq \int_{M} \sigma^{2}\left(4|\nabla v|^{2}+S_{g} v^{2}\right) d \mu-\frac{m}{2} \ln \sigma^{2}+4\left(t+\sigma^{2}\right) B A_{0}^{-1}-\frac{m}{2} \ln \frac{n A_{0}}{8 e}$.

Choosing $\beta_{1}=4 B A_{0}^{-1}=4 A_{0}^{-1}\left(B_{0}+A \sup S_{g}^{-}(x, 0)\right)$ and $A=A_{0} / 4$, we obtain the result. We can also derive (5-6) in a similar manner.

\section{Heat kernel bound via log-Sobolev inequalities}

We apply the logarithmic Sobolev inequality obtained in the last section to derive an upper bound for the conjugate heat kernel along the Ricci flow, demonstrating that there is a lot of geometric information embedded in such inequalities. The basic ideas, due to Davies and Simon [1984], relate Nelson's hypercontractivity (see [Gross 1975]) to ultracontractivity (see also [Davies 1989]). These ideas always yield estimates with sharp constants. We modify the argument in [Zhang 2007] (see also [Lieb and Loss 1997; Zhang 2011]) to prove our result. 
Theorem 6.1. Suppose there exists a solution to the $(\mathrm{RH})_{\alpha}-$ flow with $m \geq 2$ and let $H(x, t ; y)$ be the fundamental solution to the conjugate heat equation

$$
\left(-\partial_{t}-\Delta+S_{g}(x, \tau)\right) w(x, \tau)=0 .
$$

Then, for some nonnegative finite constant $C$ depending on $n, t, T, A_{0}, B_{0}$ and $\sup S_{g}^{-}(\cdot, 0)$, the estimate

$$
H(x, T ; y) \leq C T^{-m / 2}
$$

holds, where $\partial_{t} \tau=-1$ and $A_{0}, B_{0}$ are as defined in the last section.

Without loss of generality, we may assume $w=w(x, t)$ to be a nonnegative solution of the conjugate heat equation (6-1) on the interval $[0, T]$, where $\partial_{t} \tau=-1$. Let $T>0$ and $r(\tau):[0, T] \rightarrow[1, \infty]$ be a continuously differentiable increasing function such that $r(0)=\infty$ and $r(T)=1$. The function $r(\tau)=T / \tau$ gives a perfect example as we shall see below.

The idea here follows from the fact that if

$$
w(x, t)=\int H(x, t ; y) w_{0}(y) d \mu(y)
$$

solves the heat equation, where $H(x, t ; y)$ is the heat kernel, then

$$
\sup _{w \neq 0} \frac{\|w(\cdot, t)\|_{\infty}}{\|w(\cdot, 0)\|_{1}}=\sup _{x, y} H(x, t ; y) .
$$

We may obtain an estimation of the time derivative for the logarithms of the quantity

$$
\|w\|_{r(t)}=\left(\int_{M}|w|^{r(t)} d \mu_{g(t)}\right)^{1 / r(t)}
$$

as follows:

$$
\int_{0}^{T} \frac{\partial}{\partial t} \ln \|w\|_{r(t)} d t=\ln \frac{\|w(\cdot, t)\|_{\infty}}{\|w(\cdot, 0)\|_{1}} .
$$

Proof. By routine computation,

$$
\begin{aligned}
& \partial_{t}\|w\|_{r(t)}= \partial_{t}\left(\int_{M}|w|^{r(t)} d \mu_{g(\tau)}\right)^{1 / r(\tau)} \\
&=-\frac{\dot{r}(\tau)}{r^{2}(\tau)}\|w\|_{r(\tau)} \ln \|w\|_{r(\tau)}^{r(\tau)}+\frac{\|w\|_{r(\tau)}^{1-r(\tau)}}{r(\tau)}\left(\dot{r}(\tau) \int_{M} w^{r(\tau)} \ln w d \mu_{g(\tau)}\right. \\
&\left.\quad+r(\tau) \int_{M}\left(w^{r(\tau)-1}\left(-\Delta w+S_{g} w\right)+w^{r(\tau)}\left(-S_{g}\right)\right) d \mu_{g(\tau)}\right) .
\end{aligned}
$$


Multiplying both sides by $r^{2}(\tau)\|w\|_{r(\tau)}^{r(\tau)}$, we have

$$
\begin{aligned}
r^{2}(\tau)\|w\|_{r(\tau)}^{r(\tau)} & \partial_{t}\|w\|_{r(t)} \\
= & -\dot{r}(\tau)\|w\|_{r(\tau)}^{r(\tau)+1} \ln \|w\|_{r(\tau)}^{r(\tau)}+r(\tau) \dot{r}(\tau)\|w\|_{r(\tau)} \int_{M} w^{r(\tau)} \ln u d \mu_{g(\tau)} \\
& +r^{2}(\tau)\|w\|_{r(\tau)} \int_{M} w^{r(\tau)-1}(-\Delta w) d \mu_{g(\tau)}+r^{2}(\tau)\|w\|_{r(\tau)} \\
& \times \int_{M} w^{r(\tau)-1}\left(S_{g} w\right) d \mu_{g(\tau)}-r(\tau)\|w\|_{r(\tau)} \int_{M} w^{r(\tau)} S_{g} d \mu_{g(\tau)} .
\end{aligned}
$$

By the application of integration by parts, we have

$$
\begin{aligned}
r^{2}(\tau)\|w\|_{r(\tau)} \int_{M} w^{r(\tau)-1}(-\Delta & w) d \mu_{g(\tau)} \\
= & r^{2}(\tau)\|u\|_{r(\tau)} \int_{M} \nabla\left(u^{r(\tau)-1}\right) \nabla w d \mu_{g(\tau)} \\
& =r^{2}(\tau)(r(\tau)-1)\|w\|_{r(\tau)} \int_{M} w^{r(\tau)-2}|\nabla w|^{2} d \mu_{g(\tau)} .
\end{aligned}
$$

Hence,

$$
\begin{aligned}
r^{2}(\tau)\|w\|_{r(\tau)}^{r(\tau)} & \partial_{t}\|w\|_{r(t)} \\
= & -\dot{r}(\tau)\|w\|_{r(\tau)}^{r(\tau)+1} \ln \|w\|_{r(\tau)}^{r(\tau)}+r(\tau) \dot{r}(\tau)\|w\|_{r(\tau)} \int_{M} w^{r(\tau)} \ln w d \mu_{g(\tau)} \\
+ & r^{2}(\tau)(r(\tau)-1)\|w\|_{r(\tau)} \int_{M} w^{r(\tau)-2}|\nabla w|^{2} d \mu_{g(\tau)} \\
& +r(\tau)(r(\tau)-1)\|w\|_{r(\tau)} \int_{M} S_{g} w^{r(\tau)} d \mu_{g(\tau)} .
\end{aligned}
$$

Further dividing both sides by $\|w\|_{r(\tau)}$, we obtain

(6-3) $r^{2}(\tau)\|w\|_{r(\tau)}^{r(\tau)} \partial_{t}\left(\ln \|w\|_{r(t)}\right)$

$$
\begin{aligned}
& =-\dot{r}(\tau)\|u\|_{r(\tau)}^{r(\tau)} \ln \|w\|_{r(\tau)}^{r(\tau)}+r(\tau) \dot{r}(\tau) \int_{M} w^{r(\tau)} \ln w d \mu_{g(\tau)} \\
& +r^{2}(\tau)(r(\tau)-1) \int_{M} w^{r(\tau)-2}|\nabla w|^{2} d \mu_{g(\tau)} \\
& \quad+r(\tau)(r(\tau)-1) \int_{M} S_{g} w^{r(\tau)} d \mu_{g(\tau)} .
\end{aligned}
$$

Using

$$
v=\frac{w^{r(\tau) / 2}}{\left\|w^{r(\tau) / 2}\right\|_{2}} \quad \Longrightarrow \quad v^{2}=\frac{w^{r(\tau)}}{\|w\|_{r(\tau)}^{r(\tau)}},
$$


we have

$$
|\nabla v|^{2}=\frac{r^{2}(\tau)}{4\|w\|_{r(\tau)}^{r(\tau)}} w^{r(\tau)-2}|\nabla w|^{2}
$$

and

$$
\ln v^{2}=\ln w^{r(\tau)}-\ln \|w\|_{r(\tau)}^{r(\tau)}
$$

Therefore,

$$
\begin{aligned}
\dot{r}(\tau) \int_{M} v^{2} \ln v^{2} d \mu_{g(\tau)} & =\dot{r}(\tau) \int_{M} \frac{w^{r(\tau)}}{\|u\|_{r(\tau)}^{r(\tau)}}\left(\ln w^{r(\tau)}-\ln \|w\|_{r(\tau)}^{r(\tau)} g\right) d \mu_{g(\tau)} \\
& =\frac{\dot{r}(\tau) r(\tau)}{\|w\|_{r(\tau)}^{r(\tau)}} \int_{M} w^{r(\tau)} \ln w^{r(\tau)} d \mu_{g(\tau)}-\dot{r} \ln \|w\|_{r(\tau)}^{r(\tau)} .
\end{aligned}
$$

Plugging these into (6-3), we arrive at

$$
\begin{array}{r}
r^{2}(\tau) \partial_{t}\left(\ln \|w\|_{r(t)}\right)=\dot{r}(\tau) \int_{M} v^{2} \ln v^{2} d \mu_{g(\tau)}+4(r(\tau)-1) \int_{M}|\nabla v|^{2} d \mu_{g(\tau)} \\
+r(\tau)(r(\tau)-1) \int_{M} R v^{2} d \mu_{g(\tau)} \\
=\dot{r}(\tau) \int_{M} v^{2} \ln v^{2} d \mu_{g(\tau)}+(r(\tau)-1) \int_{M}\left(4|\nabla v|^{2}+S_{g} v^{2} d \mu_{g(\tau)}\right. \\
+(r(\tau)-1)^{2} \int_{M} S_{g} v^{2} d \mu_{g(\tau)} .
\end{array}
$$

Using the choice $r(\tau)=T / \tau$, we have $\dot{r}(\tau)=-T / \tau^{2}$ and $r(\tau)-1=(T-\tau) / \tau$ so that we write the last equality as

$$
\begin{array}{r}
r^{2}(\tau) \partial_{t}\left(\ln \|w\|_{r(t)}\right)=-\frac{T}{\tau^{2}} \int_{M} v^{2} \ln v^{2} d \mu_{g(\tau)}+\frac{T-\tau}{\tau} \int_{M}\left(4|\nabla v|^{2}+S_{g} v^{2}\right) d \mu_{g(\tau)} \\
+\left(\frac{T-\tau}{\tau}\right)^{2} \int_{M} S_{g} v^{2} d \mu_{g(\tau)} \\
=\frac{T}{\tau^{2}}\left(\frac{\tau(T-\tau)}{T} \int_{M}\left(4|\nabla v|^{2}+S_{g} v^{2}\right) d \mu_{g(\tau)}-\int_{M} v^{2} \ln v^{2} d \mu_{g(\tau)}\right) \\
+\left(\frac{T-\tau}{\tau}\right)^{2} \int_{M} S_{g} v^{2} d \mu_{g(\tau)} .
\end{array}
$$

From the log-Sobolev inequality (5-5) point of view, we may choose

$$
\sigma^{2}=\frac{4 \tau(T-\tau)}{T} \leq \frac{T}{4}
$$


and we get

(6-4) $\quad r^{2}(\tau) \partial_{t}\left(\ln \|w\|_{r(t)}\right)$

$$
\geq \frac{T}{\tau^{2}}\left(\frac{m}{2} \ln \sigma^{2}-\frac{m}{2} \ln \frac{m A}{2 e}-\left(t_{0}+\sigma^{2}\right) \beta_{1}\right)+\left(\frac{T-\tau}{\tau}\right)^{2} \int_{M} S_{g} v^{2} d \mu_{g(\tau)}
$$

and

(6-5) $\quad \partial_{t}\left(\ln \|w\|_{r(t)}\right)$

$$
\geq \frac{1}{T}\left(\frac{m}{2} \ln \frac{4 \pi \tau(T-\tau)}{T}-\frac{m}{2} \ln \frac{m \pi A}{2 e}-\left(t_{0}+\sigma^{2}\right) \beta_{1}-T \sup S_{g}^{-}(\cdot, 0)\right) .
$$

Notice that (since $\sigma^{2} \leq T / 4$ )

$$
\begin{aligned}
\left(t+\sigma^{2}\right) \beta_{1}+T \sup R^{-}(\cdot, 0) & =4\left(t_{0}+\sigma^{2}\right)\left(A_{0}^{-1} B_{0}+\frac{1}{4} \sup S_{g}^{-}(\cdot, 0)\right)+T \sup S_{g}^{-}(\cdot, 0) \\
& \leq\left(4 t_{0}+T\right) A_{0}^{-1} B_{0}+\frac{1}{4}\left(4 t_{0}+5 T\right) \sup S_{g}^{-}(\cdot, 0) .
\end{aligned}
$$

Denoting $D$ by

$$
D \equiv \frac{m}{2} \ln \frac{m \pi A}{2 e}+\left(4 t_{0}+T\right) A_{0}^{-1} B_{0},
$$

substituting into (6-5) and integrating the result from 0 to $T$, we have

$$
\begin{aligned}
\ln \frac{\|w(\cdot, T)\|_{r(T)}}{\|w(\cdot, T)\|_{r(0)}} & \geq \frac{m}{2 T} \int_{0}^{T} \ln \frac{4 \pi \tau(T-\tau)}{T} d t-D-\frac{1}{4}\left(4 t_{0}+5 T\right) \sup R^{-}(\cdot, 0) \\
& =\frac{m}{2} \ln (4 \pi)-\frac{n}{2} \ln T-n+n \ln T-D-\frac{1}{4}\left(4 t_{0}+5 T\right) \sup S_{g}^{-}(\cdot, 0) \\
& =\frac{m}{2} \ln (4 \pi T)-m-D-\left(4 t_{0}+5 T\right) \sup S_{g}^{-}(\cdot, 0) .
\end{aligned}
$$

This then yields

$$
\ln \frac{\|w(\cdot, T)\|_{1}}{\|w(\cdot, T)\|_{\infty}} \geq \frac{m}{2} \ln (4 \pi T)-m-D-\frac{1}{4}\left(4 t_{0}+5 T\right) \sup S_{g}^{-}(\cdot, 0),
$$

which implies

$$
\|w(\cdot, T)\|_{\infty} \leq\|w(\cdot, T)\|_{1} \frac{\exp \left(\frac{1}{4}\left(4 t_{0}+5 T\right) \sup S_{g}^{-}(\cdot, 0)+D+m\right)}{(4 \pi T)^{m / 2}} .
$$

Because

$$
w(x, T)=\int_{M} H(x, T ; y) w(y, 0) d \mu(y)_{g(\tau)},
$$

where $H(x, T ; y)$ is the conjugate heat kernel,

$$
H(x, T ; y) \leq \frac{\exp (m D)}{(4 \pi T)^{m / 2}} \exp \left(\frac{1}{4}\left(4 t_{0}+5 T\right) \sup S_{g}^{-}(\cdot, 0)\right) .
$$

This ends the proof of the estimate (6-2). 


\section{The Sobolev inequality along $(\mathrm{RH})_{\alpha}$-flow}

In this section we show that global bounds on the heat kernel to the conjugate heat equation imply a uniform Sobolev inequality under Ricci-harmonic map flow. This type of proof is standard as contained in [Davies 1989, Chapter 2]. The same procedures have been adapted in [Zhang 2007] for Kähler-Ricci flow; see also [Ye 2007; Hsu 2008]. For completeness we give the summary of the approach.

For any $t \in[0, T)$, we define the operator

$$
L:=-\Delta_{g}+\frac{S_{g}+\sup _{M} S_{g}^{-}}{4} .
$$

Since $R_{g}(\cdot, \tau) \geq-\sup _{M} R_{g}(\cdot, \tau)$, we know that $\Phi=\frac{1}{4}\left(S_{g}+\sup _{M} S_{g}^{-}\right) \geq 0$, $\Phi \in L^{\infty}(M)$. Then $L \geq 0$ and is essentially a self-adjoint operator on $L^{2}(M)$ with the associated quadratic form

$$
\boldsymbol{Q}(v)=\int_{M}\left(|\nabla v|^{2}+\Phi v^{2}\right) d \mu_{g} \quad \forall v \in W^{1,2}(M) .
$$

By the heat kernel convolution property, we have

$$
e^{-t L} w_{0}=\int_{M} H(x, t ; y) w_{0}(y) d \mu_{g}(y),
$$

where $e^{-t L}$ is a self-adjoint positivity preserving semigroup for all $t \geq 0$. It is also a contraction on $L^{\infty}(M)$ and $L^{1}(M)$ for all $t \geq 0$. Then

$$
\left\|e^{-t L} w_{0}\right\|_{\infty} \leq C_{0} t^{-m / 2}\left\|w_{0}\right\|_{1} .
$$

The next step is to apply a theorem in [Davies 1989], which we state below as a lemma.

Lemma 7.1. If $m \geq 2$, then a bound of the form

$$
\left\|e^{-t L} w_{0}\right\|_{\infty} \leq C_{1} t^{-m / 4}\left\|w_{0}\right\|_{2}
$$

for all $t>0$ and all $w_{0} \in L^{2}(M)$ is equivalent to a bound of the form

$$
\left\|w_{0}\right\|_{2 m /(m-2)}^{2} \leq C_{2} \boldsymbol{Q}\left(w_{0}\right) \quad \forall w_{0} \in W^{1,2}(M) .
$$

By Lemma 7.1 we can prove that

$$
\left(\int_{M} v^{2 m /(m-2)} d \mu_{g}\right)^{(m-2) / 2} \leq A_{0} \int_{M}\left(|\nabla v|^{2}+\frac{1}{4}\left(S_{g}+\sup _{M} S_{g}^{-}\right) v^{2}\right) d \mu_{g}
$$

using an estimate of the form (1-9). The only thing remaining for us to show is that estimates (7-4) and (7-5) are equivalent. We do this via the following lemma and the Hölder inequality. 
Lemma 7.2. Suppose $m \geq 2$ and $T<\infty$. Let $C_{1}>0$ be the same as $C_{1}$ in (7-5). Then we have

$$
\left\|e^{-t L} w_{0}\right\|_{2} \leq C_{1} t^{-m / 4}\left\|w_{0}\right\|_{1} \quad \forall w_{0} \in L^{1}(M) .
$$

Now write $e^{-t L} w_{0}=e^{-1 / 2 t L} e^{-1 / 2 t L} w_{0}$ and by assuming (7-5), we have

$$
\left\|e^{-t L} w_{0}\right\|_{\infty} \leq C_{1} t^{-m / 4}\left\|e^{-1 / 2 t L} w_{0}\right\|_{2} \leq C_{1}^{2} t^{-m / 2}\left\|w_{0}\right\|_{1} .
$$

Similarly, combining the fact that $e^{-t L}$ is a contraction on $L^{\infty}(M)$ with bound (7-4) gives us (7-5). Indeed,

$$
\begin{aligned}
\left\|e^{-t L} w_{0}\right\|_{\infty} & =\left|\int_{M} H(x, t ; y) w_{0}(y) d \mu_{g}(y)\right| \\
& \leq\left(\int_{M} H^{q^{\prime}}(x, t ; y) \mu_{g}(y)\right)^{1 / q^{\prime}}\left(\int_{M} w_{0}^{q} \mu_{g}(y)\right)^{1 / q} \leq C t^{-m / 2 q}\left\|w_{0}\right\|_{q},
\end{aligned}
$$

for all $w_{0} \in L^{q}(M)$ with $1 / q=1-1 / q^{\prime}$ and $\int_{M} H(x, t: y) d \mu_{g} \leq 1$. Here we take $q$ to satisfy $1 \leq q<m$ for obvious reason. (Though, by the Riez-Thorin interpolation theorem, the above holds for any $1 \leq q<\infty$ since $e^{-t L}$ is a contraction on $L^{1}(M)$ and $L^{\infty}(M)$.)

The main result of this section is as follows.

Theorem 7.3. With the conditions of the theorem in the introduction, we claim that estimate (1-8) implies the uniform Sobolev inequality (1-7).

Proof. Based on the previous argument and a modification of the calculation in [Zhang 2007], we define the operator $\widetilde{L}=L+1$, which also has all the properties of $L,(\widetilde{L} \geq 0$ and generates a symmetric Markov semigroup). Then for any positive constant $c$ depending on $m, T$, a lower bound for $R_{g_{0}}$ and an upper bound for $A_{0}$ such that for all $t \in[0, T)$ and $v \in \operatorname{Dom}(\widetilde{L}) \subseteq W^{1, q}(M)$,

$$
\left\|\widetilde{L}^{-1 / 2} w\right\|_{m q /(m-q)} \leq c\|w\|_{q} \quad \forall w \in W_{0}^{1,2}(M)
$$

holds for $m \geq 3$. Since $\widetilde{L}^{-1 / 2}$ is of weak type $(p, q), p=m q /(m-q)$ for any $1<q<m$. A simple analysis and the Marcinkiewicz interpolation theorem tell us that $\widetilde{L}^{-1 / 2}$ is a bounded operator from $L^{q}$ to $L^{p}$ and that (7-9) holds.

Define $v(x, t)=\widetilde{L}^{-1 / 2} w(x, t)$, which implies $w(x, t)=\widetilde{L}^{1 / 2} v(x, t)$. Taking $q=2$, we have

$$
\|w\|_{2}^{2}=\int_{M} \widetilde{L}^{1 / 2} v \widetilde{L}^{1 / 2} v d \mu_{g}=\int_{M}(\widetilde{L} v) v d \mu_{g}=\int_{M}((L+1) v) v d \mu_{g} .
$$

Combining with (7-9) and (7-6), we obtain the Sobolev inequality

$$
\|v\|_{2 m /(m-2)}^{2} \leq c C_{2}\left(\boldsymbol{Q}(v)+\int_{M} v^{2} \mu_{g}\right),
$$

whereby (1-7) follows with $A=c C_{2}$ and $B=\frac{1}{4} c C_{2}\left(\sup _{M} S_{g}+1\right)$. 
Remark 7.4. Fixing $t_{0}$ during $(\mathrm{RH})_{\alpha}$-flow, it is clear that $\widetilde{H}=e^{-1} H$ is the heat kernel of $\widetilde{L}$ and that

$$
\int_{M} \widetilde{H}(x, t ; y) d \mu_{g}(y) \leq \int_{M} H(x, t ; y) d \mu_{g}(y) \leq 1 .
$$

By the upper bound for $H$, we are sure that $\widetilde{H}$ obeys the global upper bound

$$
\widetilde{H}(x, t ; y) d \mu_{g}(y) \leq \widetilde{C} t^{-m / 2}, \quad t>0,
$$

where $\widetilde{C}$ depends on $m, A_{0}, B_{0}, t_{0}$ and $T$. Similarly,

$$
\left\|e^{-t \widetilde{L}} w\right\|_{\infty}=\left\|e^{-t} e^{-t \widetilde{L}}\right\|_{\infty} \leq e^{-t} C t^{-m / 2}\|w\|_{1}=\widetilde{C} t^{-m / 2}\|w\|_{1} .
$$

As a corollary, suppose

$$
\lambda_{\alpha 0}=\inf _{\|v\|_{2}=1} \int_{M}\left(4|\nabla v|^{2}+S_{g} v^{2}\right) d \mu_{g_{0}}>0 .
$$

It can be proved by following [Zhang 2007] that Sobolev inequality (5-2) holds with $B=B(t=0)=0$ on a compact manifold $\left(M, g_{0}\right)$; i.e.,

$$
\left(\int_{M} v^{2 m /(m-2)} d \mu_{g_{0}}\right)^{(m-2) / m} \leq \widetilde{A}_{0} \int_{M}\left(4|\nabla v|^{2}+S_{g} v^{2}\right) d \mu_{g_{0}},
$$

where $\widetilde{A}$ depends only on $m, g_{0}$ and $\lambda_{\alpha 0}$. Therefore, we have the following result.

Corollary 7.5. Let $(M, g)$ be a compact Riemannian manifold of dimension $m \geq 3$ and the metric $g(t)$ evolved by the $(\mathrm{RH})_{\alpha}$-flow. Assume that $L^{2}{ }^{2}$-Sobolev embedding (7-11) holds true with respect to the initial metric $g(0)=g_{0}$. Then, there exists a positive constant $\widetilde{A}$ depending on $\widetilde{A}_{0}$ such that for all $v \in W^{1,2}(M, g(t)), t \in[0, T)$,

$$
\left(\int_{M} v^{2 m /(m-2)} d \mu_{g(t)}\right)^{(m-2) / m} \leq \tilde{A} \int_{M}\left(4|\nabla v|^{2}+S_{g} v^{2}\right) d \mu_{g(t)},
$$

and

$$
\int_{M} v^{2} \ln v^{2} d \mu_{g(t)} \leq \sigma^{2} \int_{M}\left(4|\nabla v|^{2}+S_{g} v^{2}\right) d \mu_{g(t)}-\frac{m}{2} \ln \sigma^{2}+\frac{m}{2} \ln \frac{m A}{2 e}
$$

where $\sigma>0$.

Remark 7.6. The smallest eigenvalue is an important quantity that gives a better understanding of the geometric nature of the underlying manifold. For instance, consider the operator semigroup $e^{-t L}$ generated by $L:=-\Delta+\Phi$, with $\Phi \in L^{\infty}(M, g)$. By spectral decomposition, we write a positive solution on $M$ as

$$
U=e^{-t L} u=\sum_{j=1}^{\infty} e^{-\lambda_{j} t} \psi_{j}\left\langle u, \psi_{j}\right\rangle_{L^{2}(M)}
$$


for $u \in L^{2}(M)$ satisfying the Cauchy problem

$$
\begin{gathered}
\frac{\partial}{\partial t}\left(e^{-t L} u\right)=-L e^{-t L} u, \\
\left.U\right|_{t=0}=u,
\end{gathered}
$$

and the eigenvalue problem $L \psi=\lambda \psi$, where $\left\{\psi_{j}\right\}_{j=1}^{\infty}$ forms a complete set of $L^{2}$-orthonormal eigenfunctions of $L$ and the corresponding eigenvalues can be arranged in a nondecreasing order $\lambda_{1} \leq \lambda_{2} \leq \cdots$, with $\lambda_{j} \rightarrow \infty$. An interested reader will find the books [Davies 1989] and [Schoen and Yau 1994] useful in this respect.

\section{Acknowledgements}

The author wishes to thank the anonymous referee and the editor for their useful comments. He acknowledges the support he received from his supervisor, Professor Ali Taheri, University of Sussex, UK. He is also indebted to Dr Mihai Băileşteanu, University of Rochester, NY, for the useful discussions he had with him when he visited the University of Rochester in February 2014. His research is supported by the Tertiary Education Trust Fund, Federal Republic of Nigeria.

\section{References}

[Aubin 1976] T. Aubin, "Problèmes isopérimétriques et espaces de Sobolev", J. Differential Geometry 11:4 (1976), 573-598. MR 56 \#6711 Zbl 0371.46011

[Băileșteanu et al. 2010] M. Băileșteanu, X. Cao, and A. Pulemotov, "Gradient estimates for the heat equation under the Ricci flow", J. Funct. Anal. 258:10 (2010), 3517-3542. MR 2011b:53153 Zbl 1193.53139

[Băileșteanu and Tran 2013] M. Băileșteanu and H. Tran, "Heat kernel estimates under the RicciHarmonic map flow", preprint, 2013. arXiv 1310.1619

[Cao and Zhang 2011] X. Cao and Q. S. Zhang, "The conjugate heat equation and ancient solutions of the Ricci flow”, Adv. Math. 228:5 (2011), 2891-2919. MR 2012k:53127 Zbl 1238.53038

[Cao et al. 2003] H. D. Cao, B. Chow, S. C. Chu, and S. T. Yau (editors), Collected papers on Ricci flow, Series in Geometry and Topology 37, International Press, Somerville, MA, 2003. MR 2006e:53002 Zbl 1108.53002

[Cheeger and Yau 1981] J. Cheeger and S. T. Yau, "A lower bound for the heat kernel", Comm. Pure Appl. Math. 34:4 (1981), 465-480. MR 82i:58065 Zbl 0481.35003

[Chow and Knopf 2004] B. Chow and D. Knopf, The Ricci flow: An introduction, Mathematical Surveys and Monographs 110, Amer. Math. Soc., Providence, RI, 2004. MR 2005e:53101 Zbl 1086.53085

[Chow et al. 2008] B. Chow, S.-C. Chu, D. Glickenstein, C. Guenther, J. Isenberg, T. Ivey, D. Knopf, P. Lu, F. Luo, and L. Ni, The Ricci flow: Techniques and applications, II: Analytic aspects, Mathematical Surveys and Monographs 144, Amer. Math. Soc., Providence, RI, 2008. MR 2008j:53114 Zbl 1157.53035 
[Davies 1989] E. B. Davies, Heat kernels and spectral theory, Cambridge Tracts in Mathematics 92, Cambridge Univ. Press, 1989. MR 90e:35123 Zbl 0699.35006

[Davies and Simon 1984] E. B. Davies and B. Simon, "Ultracontractivity and the heat kernel for Schrödinger operators and Dirichlet Laplacians", J. Funct. Anal. 59 (1984), 335-395. MR 86e:47054 Zbl 0568.47034

[Eells and Sampson 1964] J. Eells, Jr. and J. H. Sampson, "Harmonic mappings of Riemannian manifolds", Amer. J. Math. 86 (1964), 109-160. MR 29\#1603 Zbl 0122.40102

[Gross 1975] L. Gross, "Logarithmic Sobolev inequalities", Amer. J. Math. 97:4 (1975), 1061-1083. MR 54 \#8263 Zbl 0318.46049

[Hamilton 1982] R. S. Hamilton, "Three-manifolds with positive Ricci curvature", J. Differential Geom. 17:2 (1982), 255-306. MR 84a:53050 Zbl 0504.53034

[Hamilton 1993] R. S. Hamilton, "The Harnack estimate for the Ricci flow", J. Differential Geom. 37:1 (1993), 225-243. MR 93k:58052 Zbl 0804.53023

[Hebey 1996] E. Hebey, "Optimal Sobolev inequalities on complete Riemannian manifolds with Ricci curvature bounded below and positive injectivity radius", Amer. J. Math. 118:2 (1996), 291-300. MR 97c:53058 Zbl 0863.53031

[Hsu 2008] S.-Y. Hsu, "Sobolev inequalities for manifolds evolving by Ricci flow", Adv. Math. Sci. Appl. 18:2 (2008), 453-462. MR 2010f:53112 Zbl 1187.53070

[Kuang and Zhang 2008] S. Kuang and Q. S. Zhang, "A gradient estimate for all positive solutions of the conjugate heat equation under Ricci flow", J. Funct. Anal. 255:4 (2008), 1008-1023. MR 2009h:53150 Zbl 1146.58017

[Li and Yau 1986] P. Li and S.-T. Yau, "On the parabolic kernel of the Schrödinger operator", Acta Math. 156:3-4 (1986), 153-201. MR 87f:58156 Zbl 0611.58045

[Lieb and Loss 1997] E. H. Lieb and M. Loss, Analysis, Graduate Studies in Mathematics 14, Amer. Math. Soc., Providence, RI, 1997. MR 98b:00004 Zbl 0873.26002

[List 2008] B. List, "Evolution of an extended Ricci flow system", Comm. Anal. Geom. 16:5 (2008), 1007-1048. MR 2010i:53126 Zbl 1166.53044

[Müller 2012] R. Müller, "Ricci flow coupled with harmonic map flow", Ann. Sci. Éc. Norm. Supér. (4) 45:1 (2012), 101-142. MR 2961788 Zbl 1247.53082

[Ni 2004] L. Ni, "The entropy formula for linear heat equation”, J. Geom. Anal. 14:1 (2004), 87-100. MR 2004m:53118a Zbl 1044.58030

[Ni 2006] L. Ni, “A note on Perelman's LYH-type inequality”, Comm. Anal. Geom. 14:5 (2006), 883-905. MR 2007h:53103 Zbl 1116.58031

[Perelman 2002] G. Perelman, "The entropy formula for the Ricci flow and its geometric application", preprint, 2002. arXiv math.DG/0211159v1

[Perelman 2003a] G. Perelman, "Finite extinction time for the solutions to the Ricci flow on certain three-manifolds", preprint, 2003. arXiv math.DG/0307245v1

[Perelman 2003b] G. Perelman, "Ricci flow with surgery on three manifolds", preprint, 2003. arXiv math.DG/0303109v1

[Schoen and Yau 1994] R. Schoen and S.-T. Yau, Lectures on differential geometry, Conference Proceedings and Lecture Notes in Geometry and Topology 1, International Press, Cambridge, MA, 1994. MR 97d:53001 Zbl 0830.53001

[Ye 2007] R. Ye, "The logarithmic Sobolev inequalities along the Ricci flow", preprint, 2007. arXiv 0707.2424v4 
[Zhang 2007] Q. S. Zhang, "A uniform Sobolev inequality under Ricci flow", Int. Math. Res. Not. 2007:17 (2007), Art. ID rnm056. MR 2008g:53083 Zbl 1141.53064

[Zhang 2011] Q. S. Zhang, Sobolev inequalities, heat kernels under Ricci flow, and the Poincaré conjecture, CRC Press, Boca Raton, FL, 2011. MR 2011m:53127 Zbl 1208.58032

Received March 7, 2014. Revised January 14, 2015.
ABIMBOLA ABOLARINWA
DEPARTMENT OF MATHEMATICS
UNIVERSITY OF SUSSEX
BRIGHTON
BN1 9QH
UNITED KINGDOM
a.abolarinwa@sussex.ac.uk 


\title{
PACIFIC JOURNAL OF MATHEMATICS
}

\author{
msp.org/pjm
}

Founded in 1951 by E. F. Beckenbach (1906-1982) and F. Wolf (1904-1989)

\section{EDITORS}

Don Blasius (Managing Editor)

Department of Mathematics

University of California

Los Angeles, CA 90095-1555

blasius@math.ucla.edu

\author{
Paul Balmer \\ Department of Mathematics \\ University of California \\ Los Angeles, CA 90095-1555 \\ balmer@math.ucla.edu \\ Robert Finn \\ Department of Mathematics \\ Stanford University \\ Stanford, CA 94305-2125 \\ finn@math.stanford.edu \\ Sorin Popa \\ Department of Mathematics \\ University of California \\ Los Angeles, CA 90095-1555 \\ popa@math.ucla.edu
}

\author{
Vyjayanthi Chari \\ Department of Mathematics \\ University of California \\ Riverside, CA 92521-0135 \\ chari@math.ucr.edu \\ Kefeng Liu \\ Department of Mathematics \\ University of California \\ Los Angeles, CA 90095-1555 \\ liu@math.ucla.edu \\ Jie Qing \\ Department of Mathematics \\ University of California \\ Santa Cruz, CA 95064 \\ qing@ cats.ucsc.edu
}

\section{PRODUCTION}

Silvio Levy, Scientific Editor, production@msp.org

\section{SUPPORTING INSTITUTIONS}

ACADEMIA SINICA, TAIPEI

CALIFORNIA INST. OF TECHNOLOGY

INST. DE MATEMÁTICA PURA E APLICADA

KEIO UNIVERSITY

MATH. SCIENCES RESEARCH INSTITUTE

NEW MEXICO STATE UNIV.

OREGON STATE UNIV.

\author{
STANFORD UNIVERSITY \\ UNIV. OF BRITISH COLUMBIA \\ UNIV. OF CALIFORNIA, BERKELEY \\ UNIV. OF CALIFORNIA, DAVIS \\ UNIV. OF CALIFORNIA, LOS ANGELES \\ UNIV. OF CALIFORNIA, RIVERSIDE \\ UNIV. OF CALIFORNIA, SAN DIEGO \\ UNIV. OF CALIF., SANTA BARBARA
}

\author{
Daryl Cooper \\ Department of Mathematics \\ University of California \\ Santa Barbara, CA 93106-3080 \\ cooper@math.ucsb.edu \\ Jiang-Hua Lu \\ Department of Mathematics \\ The University of Hong Kong \\ Pokfulam Rd., Hong Kong \\ jhlu@maths.hku.hk \\ Paul Yang \\ Department of Mathematics \\ Princeton University \\ Princeton NJ 08544-1000 \\ yang@math.princeton.edu
}

These supporting institutions contribute to the cost of publication of this Journal, but they are not owners or publishers and have no responsibility for its contents or policies.

See inside back cover or msp.org/pjm for submission instructions.

The subscription price for 2015 is US \$420/year for the electronic version, and \$570/year for print and electronic.

Subscriptions, requests for back issues and changes of subscribers address should be sent to Pacific Journal of Mathematics, P.O. Box 4163, Berkeley, CA 94704-0163, U.S.A. The Pacific Journal of Mathematics is indexed by Mathematical Reviews, Zentralblatt MATH, PASCAL CNRS Index, Referativnyi Zhurnal, Current Mathematical Publications and Web of Knowledge (Science Citation Index).

The Pacific Journal of Mathematics (ISSN 0030-8730) at the University of California, c/o Department of Mathematics, 798 Evans Hall \#3840, Berkeley, CA 94720-3840, is published twelve times a year. Periodical rate postage paid at Berkeley, CA 94704, and additional mailing offices. POSTMASTER: send address changes to Pacific Journal of Mathematics, P.O. Box 4163, Berkeley, CA 94704-0163.

PJM peer review and production are managed by EditFLOW ${ }^{\circledR}$ from Mathematical Sciences Publishers.

\section{PUBLISHED BY}

\section{mathematical sciences publishers \\ nonprofit scientific publishing}

http://msp.org/

(C) 2015 Mathematical Sciences Publishers 


\section{PACIFIC JOURNAL OF MATHEMATICS}

Volume $278 \quad$ No. $2 \quad$ December 2015

Differential Harnack and logarithmic Sobolev inequalities along

Ricci-harmonic map flow

ABIMBOLA ABOLARINWA

On $J$-holomorphic curves in almost complex manifolds with

291

asymptotically cylindrical ends

ERKAO BAO

Integration of coupling Dirac structures

OLIVIER BRAHIC and RUI LOJA FERNANDES

Asymptotic behavior of Palais-Smale sequences associated with fractional Yamabe-type equations

Yi FANG and MARÍA DEL MAR GONZÁLEZ

$K$-theory and homotopies of 2-cocycles on higher-rank graphs

ELIZABETH GILLASPY

Fusion products and toroidal algebras

DENIZ KUS and PETER LiTTELMANN

Differential Harnack estimates for positive solutions to heat equation under Finsler-Ricci flow

\section{SAJJAD LAKZIAN}

On the one-endedness of graphs of groups

NichOLAS TOUIKAN

On the structure of vertex cuts separating the ends of a graph

GARETH R. WILKES 